OPEN ACCESS

Edited by:

Katiucia Batista Silva Paiva,

University of São Paulo, Brazil

Reviewed by:

Maria Fernanda Forni,

Yale University, United States

Luena Papa,

Icahn School of Medicine at Mount

Sinai, United States

José Manuel García-Heredia,

University of Seville, Spain

*Correspondence.

Bin Cheng

chengbin@mail.sysu.edu.cn

Juan Xia

xiajuan@mail.sysu.edu.cn

tThese authors have contributed equally to this work

Specialty section:

This article was submitted to

Stem Cell Research,

a section of the journal

Frontiers in Cell and Developmental

Biology

Received: 04 July 2020

Accepted: 24 September 2020

Published: 15 October 2020

Citation:

Ren L, Chen X, Chen X, Li J,

Cheng B and Xia J (2020)

Mitochondrial Dynamics: Fission and Fusion in Fate Determination

of Mesenchymal Stem Cells.

Front. Cell Dev. Biol. 8:580070.

doi: 10.3389/fcell.2020.580070

\section{Mitochondrial Dynamics: Fission and Fusion in Fate Determination of Mesenchymal Stem Cells}

\author{
Lin Ren 1,2,3†, Xiaodan Chen ${ }^{1,2,3 \dagger}$, Xiaobing Chen ${ }^{1,2,3}$, Jiayan Li1,2,3, Bin Cheng ${ }^{1,2,3 *}$ and \\ Juan Xia ${ }^{1,2,3 *}$ \\ ${ }^{1}$ Hospital of Stomatology, Sun Yat-sen University, Guangzhou, China, ${ }^{2}$ Guangdong Provincial Key Laboratory \\ of Stomatology, Guangzhou, China, ${ }^{3}$ Guanghua School of Stomatology, Sun Yat-sen University, Guangzhou, China
}

Mesenchymal stem cells (MSCs) are pivotal to tissue homeostasis, repair, and regeneration due to their potential for self-renewal, multilineage differentiation, and immune modulation. Mitochondria are highly dynamic organelles that maintain their morphology via continuous fission and fusion, also known as mitochondrial dynamics. MSCs undergo specific mitochondrial dynamics during proliferation, migration, differentiation, apoptosis, or aging. Emerging evidence suggests that mitochondrial dynamics are key contributors to stem cell fate determination. The coordination of mitochondrial fission and fusion is crucial for cellular function and stress responses, while abnormal fission and/or fusion causes MSC dysfunction. This review focuses on the role of mitochondrial dynamics in MSC commitment under physiological and stress conditions. We highlight mechanistic insights into modulating mitochondrial dynamics and mitochondrial strategies for stem cell-based regenerative medicine. These findings shed light on the contribution of mitochondrial dynamics to MSC fate and MSC-based tissue repair.

Keywords: mesenchymal stem cells, mitochondria, mitochondrial dynamics, mitochondrial fission, mitochondrial fusion, cell fate

\section{INTRODUCTION}

Mesenchymal stem cells (MSCs) are multipotent stromal cells that originate from many connective tissues and can differentiate into a variety of cell types, such as osteoblasts, adipocytes, and myoblasts. MSCs are important for preserving tissue homeostasis and have regeneration potential (Bianco, 2014). As the progenitor cells of osteoblasts and osteocytes, MSCs can migrate to defective sites and initiate new bone formation during the early stage of bone healing (Tang et al., 2009). In addition to osteoblasts, MSCs are also able to differentiate into adipocytes within bone marrow microenvironment. Increasing evidence suggests that the differentiation of MSCs into adipocytes or osteoblasts is competitively balanced (Li et al., 2016), and this delicate

Abbreviations: MSCs, mesenchymal stem cells; Drp1, dynamin-related protein 1; Mff, mitochondrial fission factor; Fis1, mitochondrial fission protein 1 ; Opa1, optic atrophy $1 ; \mathrm{Mfn} 1 / 2$, mitofusin $1 / 2 ; \mathrm{ROS}$, reactive oxygen species; MAPKs, mitogenactivated protein kinases; JNK, c-Jun N-terminal kinase; ERK, extracellular regulated kinase; iPSCs, induced pluripotent stem cells; AMPK, adenosine monophosphate-activated protein kinase; IO, iron overload; BMSCs, bone marrow mesenchymal stem cells; ASCs, adipose-derived mesenchymal stem cells; OXPHOS, oxidative phosphorylation; ESCs, embryonic stem cells; HSCs, hematopoietic stem cells; NSCs, embryonic mouse neural stem cells; EMS, equine metabolic syndrome. 
balance is important for the maintenance of bone homeostasis (Hu et al., 2018b; Muruganandan et al., 2020). Dysregulation of osteo-adipogenic differentiation balance of MSCs contributes to development of bone diseases, such as osteoporosis, which manifests typically as a lineage shift toward adipocytes instead of osteoblasts in MSCs (Qi et al., 2017). Moreover, MSCs play a key role in the bone marrow microenvironment, which supports and regulates the stem cell niche and hematopoiesis (Wu et al., 2018). In recent years, MSCs have emerged as a promising tool for tissue repair and regeneration due to their multilineage differentiation potential, angiogenesis promotion, and immunomodulatory capacity (Chamberlain et al., 2007; Rajabzadeh et al., 2019). However, the effectiveness of MSCs is unstable since MSC fate is easily affected by the surrounding microenvironment, involving a complex regulation network (Sisakhtnezhad et al., 2017), and both endogenous and exogenous MSCs inevitably face harsh surrounding conditions and oxidative stress around the defected tissues (Chen et al., 2016; Sisakhtnezhad et al., 2017). Understanding the etiology of MSC dysfunction under stress and the underlying mechanisms would uncover unique avenues for novel and effective therapeutic strategies in MSCbased regenerative medicine.

Mitochondria are highly dynamic organelles that are key players in various biological processes in stem cells, including energy metabolism, oxidative stress reaction, calcium balance, and cell apoptosis. Dynamic changes in mitochondrial morphology are the basis for mitochondrial functionality (Chen and Chan, 2005; El-Hattab et al., 2018). Mitochondrial dynamics involves continuous fission and fusion, forming a dynamic network to maintain their abundance, morphology, and quality and cell function (Bereiter-Hahn and Vöth, 1994; Merz et al., 2007). This dynamic change in mitochondria can typically be simply characterized by morphological heterogeneity. Specifically, mitochondrial fission results in small and round mitochondria, while mitochondrial fusion leads to thin and elongated mitochondria with highly interconnected networks (Santel and Fuller, 2001; Stiles and Shirihai, 2012). Mitochondrial fission is essential for cell growth and division, providing sufficient numbers of mitochondria, sustaining cell polarity, and aiding in eliminating damaged mitochondria (Wai and Langer, 2016). In contrast, mitochondrial fusion allows for the exchange and connection of mitochondrial content, providing sufficient energy, alleviating oxidative damage, and maintaining membrane potential (Ikeda et al., 2015).

Although still in its infancy, emerging evidence indicates a pivotal role of mitochondrial dynamics in the self-renewal, differentiation, and death of MSCs. Mitochondrial dynamics are critical for MSCs to acquire the mitochondrial morphology required for specific behavioral needs, enabling cells to respond quickly and adaptively to environmental stresses. Intervention in mitochondrial dynamics can profoundly affect the MSC fate. In the current review, we illustrate the key mechanisms related to the posttranslational modification of mitochondrial dynamics proteins. Then, we reveal typical characteristics of mitochondria in MSCs and the contribution of mitochondrial dynamics to orchestrating MSC behavior under physiological and stressful microenvironments. Furthermore, we discuss the potential strategies for improving the therapeutic efficacy of MSCs by the modulation of mitochondrial dynamics.

\section{REGULATORY MECHANISMS OF MITOCHONDRIAL DYNAMICS}

Mitochondrial fission and fusion are orchestrated by a series of evolutionarily conserved proteins and dynamin-related GTPases. The large GTPase dynamin-related protein 1 (Drp1) and mitochondrial outer membrane receptors, including mitochondrial fission factor (Mff), mitochondrial fission protein 1 (Fis1) and mitochondrial dynamics proteins of 51 and $49 \mathrm{kD}$ (MiD51 and MiD49), prominently control mitochondrial fission (Losón et al., 2013). Mitochondrial fusion is mainly mediated by optic atrophy 1 (Opa1) located on the mitochondrial outer membrane and mitofusin 1/2 (Mfn1/2) located on the inner membrane (Santel and Fuller, 2001; Chen and Chan, 2005). Here we list some protein kinase pathways that induce posttranslational modification of these proteins, especially phosphorylation, to further elucidate the regulatory mechanisms of mitochondrial dynamics (Table $\mathbf{1}$ ).

\section{cAMP-Dependent Protein Kinase (PKA)}

The ubiquitous second messenger PKA, one of the most wellinvestigated cytosolic kinases, is located on the mitochondrial surface and plays a regulatory role in maintaining mitochondrial activity, including mitochondrial dynamics (Feliciello et al., 2005; Cribbs and Strack, 2007). Drp1 relies on a GTP hydrolysisdependent mechanism to form ring superstructures to contract and eventually incise mitochondria. Drp1 Serine 656 (Ser656) of Drp1 is a major PKA phosphorylation site in rat PC12 cells, this phosphorylation inhibits mitochondrial fission and reduces cellular sensitivity to apoptotic stimuli. Although Ser656 phosphorylation site is near the GTPase effector domain, there is no significant change in GTP hydrolysis among the Ser656 variants (Cribbs and Strack, 2007). However, in HeLa cells, active PKA suppresses Drp1 GTPase activity via Ser637 phosphorylation of Drp1, which may favor GTPase inactivation and Drp1 localization in the cytoplasm rather than recruitment to mitochondria, dampening mitochondrial fission (Chang and Blackstone, 2007; Yu et al., 2019). In contrast, phosphorylation of Drp1 at Ser600 is also dependent on adrenergic-stimulated PKA activation, inducing increased mitochondrial fragmentation and energy expenditure in brown adipocytes (Wikstrom et al., 2014). These findings suggest that $\mathrm{cAMP} / \mathrm{PKA}$ controls mitochondrial fission principally via Drp1 modulation, and its effect on fission depends on phosphorylation sites and Drp1 transport. In fact, post-translational phosphorylation of Drp1 predominantly affects Drp1 activity. The different phosphorylated sites of Drp1 and they mediated-mitochondrial fission are demonstrated in Figure 1.

Some evidence has confirmed the role of the cAMP/PKA pathway in influencing mitochondrial fusion-related proteins. In myoblasts stimulated by reactive oxygen species (ROS), decreased mitochondrial cAMP/PKA signaling induces Sirt3 degradation/proteolysis, which in turn promotes 
TABLE 1 | Post-translational modification of some key factors involved in mitochondrial dynamics.

\begin{tabular}{|c|c|c|c|}
\hline Key factor & Post-translational modification & Effect on mitochondrial dynamics & References \\
\hline \multirow[t]{9}{*}{ Drp1 } & Phosphorylation at Ser656 induced by active PKA & Reduced mitochondrial fission and swollen mitochondria & Cribbs and Strack, 2007 \\
\hline & Phosphorylation at Ser637 induced by active PKA & $\begin{array}{l}\text { Reduced GTPase activity of Drp1 and impaired mitochondrial } \\
\text { fission }\end{array}$ & $\begin{array}{l}\text { Chang and Blackstone, } \\
2007\end{array}$ \\
\hline & Phosphorylation at Ser600 induced by active PKA & Enhanced mitochondrial fission under norepinephrine treatment & Wikstrom et al., 2014 \\
\hline & Phosphorylate at Ser616 by induced by active ERK2 & Enhanced mitochondrial fission & Kashatus et al., 2015 \\
\hline & Phosphorylation of at Ser579 induced by active ERK1/2 & Enhanced mitochondrial fission in early stage of reprogramming & Prieto et al., 2016 \\
\hline & Phosphorylation at Ser616 induced by active p38 MAPK & Enhanced mitochondrial fission & Ko et al., 2017 \\
\hline & Phosphorylation at Ser616 induced by active AMPK & Enhanced mitochondrial fission & Li et al., 2019 \\
\hline & Phosphorylation at Ser616 induced by SIRT4 depletion & $\begin{array}{l}\text { Increased Drp1 and Fis-1combination, enhanced mitochondrial } \\
\text { fission }\end{array}$ & Fu et al., 2017 \\
\hline & Phosphorylation at Ser637 induced by active SIRT5 & Reduced mitochondrial fission under starvation & Guedouari et al., 2017 \\
\hline \multirow[t]{2}{*}{ Mff } & Phosphorylation at Ser155 induced by active AMPK & $\begin{array}{l}\text { Increased Drp1 recruitment, upregulated pSer616-Drp1, } \\
\text { enhanced mitochondrial fission }\end{array}$ & Zheng et al., 2018 \\
\hline & Phosphorylation at Ser155, 172 induced by active AMPK & $\begin{array}{l}\text { Increased Drp1 recruitment to mitochondria, enhanced } \\
\text { mitochondrial fission }\end{array}$ & Toyama et al., 2016 \\
\hline \multirow[t]{2}{*}{ Mfn1 } & Phosphorylation at Thr562 induced by active ERK2 & $\begin{array}{l}\text { Regulates Mfn1 oligomerization, increased Mfn1 combination with } \\
\text { Bak, reduced mitochondrial fusion }\end{array}$ & Pyakurel et al., 2015 \\
\hline & Phosphorylation at Ser86 induced by beta II PKC & $\begin{array}{l}\text { Partial inactivation of Mfn1 GTPase, increased mitochondria } \\
\text { fragmentation }\end{array}$ & Ferreira et al., 2019 \\
\hline \multirow[t]{3}{*}{ Mfn2 } & Phosphorylation at Ser442 induced by active PKA & Extensive perinuclear mitochondria & Zhou et al., 2010 \\
\hline & Phosphorylation at Ser27 induced by active JNK & $\begin{array}{l}\text { Ubiquitin-proteasome degradation in Mfn2 and reduced } \\
\text { mitochondrial fusion }\end{array}$ & Leboucher et al., 2012 \\
\hline & Ser-phosphorylation induced by active JNK & Mfn2 degradation and reduced mitochondrial fusion & Chakraborty et al., 2018 \\
\hline \multirow[t]{4}{*}{ Opa1 } & $\begin{array}{l}\text { Acetylation induced by cAMP/PKA mediated-degradation } \\
\text { Sirt3 }\end{array}$ & $\begin{array}{l}\text { Proteolytic Opa1, inhibited mitochondrial fusion under tert-butyl } \\
\text { hydroperoxide treatment }\end{array}$ & Signorile et al., 2017 \\
\hline & Deacetylation at Lys926 and 931 induced by active SIRT3 & Enhanced mitochondrial fusion, sustain mitochondrial network & Samant et al., 2014 \\
\hline & Proteolytic induced by active SIRT4 & Upregulation of L-Opa1, enhanced mitochondrial fusion & Lang et al., 2017 \\
\hline & Acetylation induced by SIRT3 deletion & $\begin{array}{l}\text { Enhanced mitochondrial fission, dramatic mitochondrial } \\
\text { fragmentation }\end{array}$ & Yi et al., 2019 \\
\hline
\end{tabular}

the hyperacetylation of Opal and short Opal generation, consequently leading to hyperfragmentation of mitochondria and cell apoptosis (Signorile et al., 2017). Treatment with 8-Br-cAMP, an analog of cAMP, reverses the detrimental effect of Opal on mitochondrial dynamics, as well as cytochrome c release under oxidative stress in cardiac myoblast cells (Signorile et al., 2017). Although Zhou et al. (2010) verified that the specific PKA phosphorylation site Ser442 of Mfn2 effectively promotes Mfn2-mediated inhibition of vascular smooth muscle cell growth, these effects are thought to be independent of mitochondrial morphology and dynamics changes.

\section{Mitogen-Activated Protein Kinases (MAPKs)}

Mitogen-activated protein kinases are serine-threonine kinases that transmit signals driven by cytokines, hormones, and other factors from the cell surface to the nucleus. At least three MAPK families have been characterized, c-Jun N-terminal kinase (JNK), extracellular regulated kinase (ERK1/2), and p38, which are involved in a wide variety of cellular functions, such as proliferation, apoptosis, and differentiation (Widmann et al., 1999). The most wellcharacterized MAPK with a confirmed regulatory effect on mitochondrial dynamics is ERK1/2 (Serasinghe et al., 2015;
Cook et al., 2017). Oncogenic Ras treatment can activate ERK2 to phosphorylate Drp1 at Ser616, resulting in an increase in mitochondrial fragmentation. Both activation of MEK activity and elevated ERK phosphorylation lead to consistent trends in the expression of Drp1 (Kashatus et al., 2015). ERK suppresses mitochondrial fusion by phosphorylating Mfn1 at threonine 562 (Thr562), which favors Mfn1 binding to BAK and promotes their oligomerization and activation, subsequently facilitating cytochrome c release and apoptosis. This indicates the significant role of ERK-targeted Mfn1 in modulating mitochondrial morphology and apoptosis for mouse embryonic fibroblasts (Pyakurel et al., 2015). ERK1/2 activation participates in mitochondrial fission mediation during the early stage of induced pluripotent stem cell (iPSC) reprogramming by phosphorylating Drp1 at Ser579. Additionally, MEK inhibitor treatment inhibits mitochondrial fragmentation, while mutations in Ser579 of Drp1 successfully rescue the fission inhibition caused by MEK inhibitor application (Prieto et al., 2016). Similarly, treatment with the MEK inhibitor, PD325901, results in dramatic decline in both ERK and Drp1616 phosphorylation, effectively reversing mitochondrial fragmented phenotype in oncogenic Ras-induced embryonic kidney cells (Kashatus et al., 2015).

JNK activation is connected to Mfn2 but not Mfn1 turnover (Chakraborty et al., 2018). Leboucher et al. demonstrated 


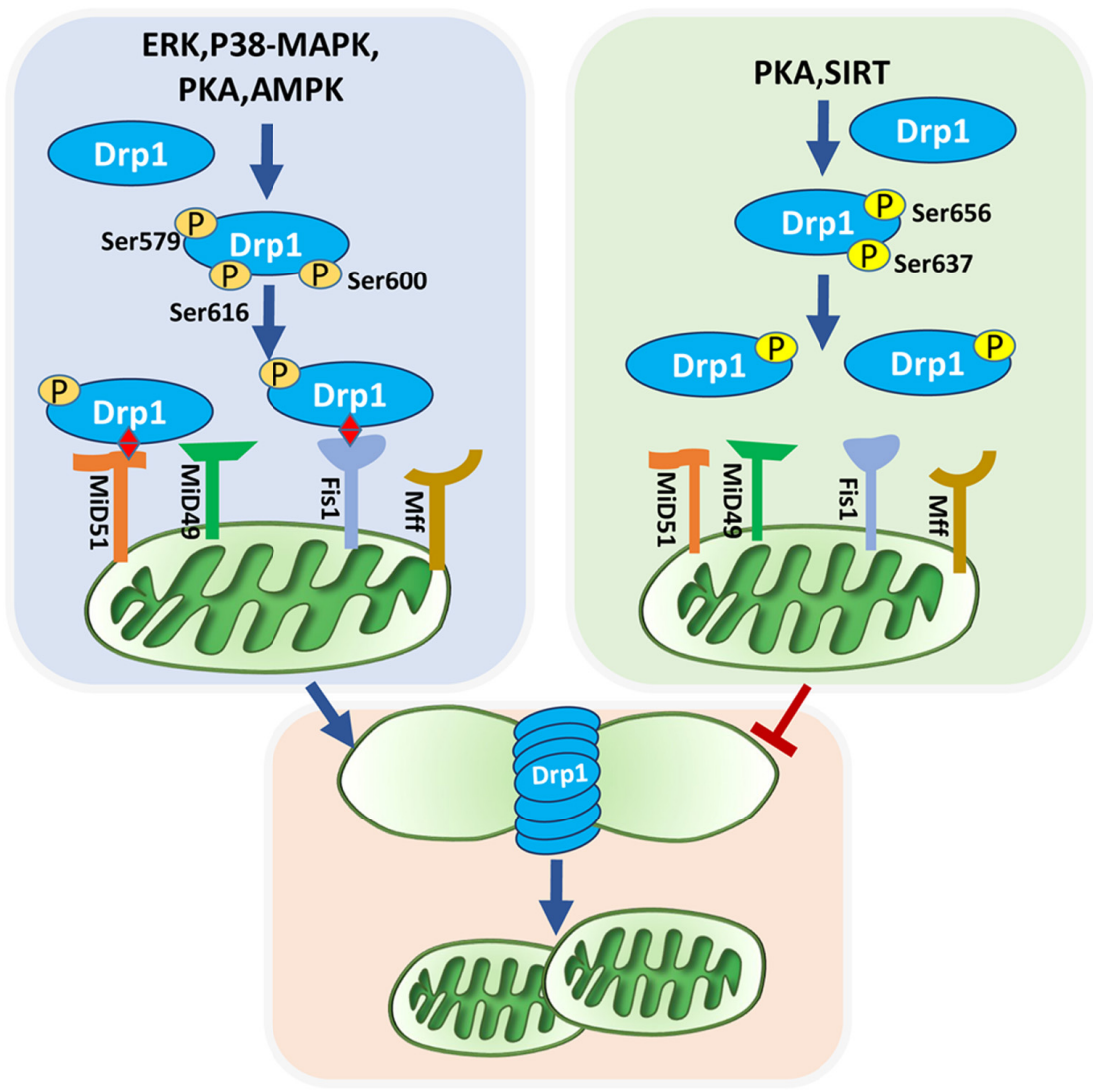

FIGURE 1 | Phosphorylated Drp1-mediated mitochondrial fission. Mitochondrial fission is mediated by recruitment of Drp1 and their anchor on the outer mitochondrial membrane. Post-translational modifications of Drp1, especially phosphorylation, affect their localization in the cytoplasm or on the outer mitochondrial membrane. ERK, P38-MAPK, PKA, AMPK, and SIRT can phosphorylate Drp1. Phosphorylation of Drp1 at Ser637 and Ser656 inhibit mitochondrial fission, whereas phosphorylation of Drp1 at Ser616, Ser579, and Ser600 promote mitochondrial fission. Drp1 is anchored to the outer mitochondrial membrane via bounding with four receptors: Fis1, Mff, Mid49, and Mid51. Active Drp1 oligomers are assembled into ring-like structures that further constrict to sever the mother mitochondria into daughter mitochondria.

that JNK mediated the phosphorylation of Mfn2 at Ser27 of sarcoma U2OS cells in response to cellular stress, which contributed to ubiquitin-proteasome degradation in Mfn2 and enhanced apoptosis (Leboucher et al., 2012). Ko et al. (2017) showed that p38 MAPK promoted Drp1-dependent fission in MSCs, and treatment with SB203580, a p38 inhibitor, reversed Drp1 phosphorylation at Ser616, leading to a decline in fragmented mitochondria.

\section{Adenosine Monophosphate-Activated Protein Kinase (AMPK)}

AMPK is a conserved, redox-activated cellular energy sensor and regulator that is sensitive to AMP/adenosine- $5^{\prime}$-triphosphate (ATP) stimulation. This kinase is activated during ATP consumption in response to stresses, such as low glucose, hypoxia, and ischemia (Carling, 2004). Reduced ATP levels induced by, for example, iron overload (IO), can activate AMPK in bone marrow mesenchymal stem cells (BMSCs), followed by Mff phosphorylation, further resulting in Drp1 translocation to mitochondria and fission enhancement, which partly contributes to BMSC dysfunction derived from IO patients with myelodysplastic syndrome (Zheng et al., 2018). The expression of p-Drp1 was downregulated while Mfn2 was upregulated in BMSCs after they were treated with compound $\mathrm{C}$, an AMPK inhibitor, and cell senescence was therefore increased. These results indicated that mitochondrial fission activated by AMPK protected BMSCs from senescence (Li et al., 2019). At low ATP levels, AMPK-mediated fission is promoted due to Drp1 transport to the mitochondrial membrane stimulated by phosphorylation of Mff (Ducommun et al., 2015; Toyama et al., 2016). Consistently, stimulation of U2OS osteosarcoma cells with either electron transport chain inhibitor complexes or AMPK agonists resulted in significantly increased mitochondrial fragmentation (Toyama et al., 2016). AMPK-mediated mitochondrial fission is widely involved in cellular reaction to energy stress, and these mitochondrial fission events may serve as a trigger to initiating mitophagy to remove damaged mitochondria (Youle and van der Bliek, 2012; Toyama et al., 2016).

In contrast, activation of AMPK by a specific AMPK activator reversed the reduction in drug-induced mitochondrial 
fusion via upregulation of Mfn1, Mfn2, and Opa1, thereby sustaining hepatocyte viability (Kang et al., 2016). AMPK activation by AICAR, a known activator of AMPK, induced fused mitochondria and interconnected networks when adiposederived mesenchymal stem cells (ASCs) from pericardial adipose tissue were differentiated into adipocytes (Abdul-Rahman et al., 2016). In addition, AMPK activated by metformin is able to suppress Drp1-dependent mitochondrial fission and alleviate oxidative stress, thus ameliorating atherosclerosis in diabetic mice (Wang et al., 2017b). However, mitochondrial fusion activated by AMPK is not always related to positive consequences. TGF-1 induces mitochondrial fusion through the AMPK signaling pathway, which induces aging of vascular progenitor cells isolated from patients with Marfan syndrome (He et al., 2019).

These findings suggest that AMPK activation can have opposing effect on mitochondrial dynamics, which possibly depend on cell types, bioenergetic status, and oxidative stress levels. The positive situation is that active AMPK sustains well-quality mitochondria at least partially via triggering mitochondrial fission and subsequent autophagy. When damage or stress is alleviated or removed, AMPK may support mitochondrial function by strengthening fusion rather than fission. Although AMPK-mediated mitochondrial dynamics have been found to be involved in some cellular damage processes, their relationship and detailed mechanisms have not been well studied.

\section{Sirtuin (Sirt) Family}

The Sirt family is composed of highly conserved nicotinamide adenine dinucleotide $\left(\mathrm{NAD}^{+}\right)$-dependent deacetylases (HDACs). There are seven mammalian sirtuin subtypes (SIRT1 to SIRT7) that have different subcellular localizations and regulate a variety of cellular functions through posttranslational modifications of target proteins (Huang et al., 2010). Among them, SIRT3, SIRT4, and SIRT5 are located on mitochondria and can mediate the activity of some proteins related to mitochondrial dynamics (Huang et al., 2010; Samant et al., 2014; Lang et al., 2017).

SIRT3-mediated deacetylation of Opal at lysine 926 and 931 enhances Opa1 GTPase activity and sustains mitochondrial morphology, protecting cardiomyocytes from doxorubicininduced cell death (Samant et al., 2014). Accurately, OPA1 appears to work in two different isoforms. Long membranebound form of OPA1 is responsible for mitochondrial fusion. But cleavage of long OPA1 inhibits mitochondrial fusion accompanied with generation of fission or mitophagy-associated short, soluble forms (MacVicar and Langer, 2016). SIRT4 expression facilitates mitochondrial fusion with an incremental increase in mitochondrial mass by inducing long Opa1 instead of short Opa1, further contributing to decreased mitophagy in HEK293 cell lines (Lang et al., 2017). In addition to promoting fusion, Fu et al. (2017) showed that SIRT4 may modulate mitochondrial fission in lung cancer cell lines by reducing Drp1 phosphorylation and diminishing Drp1 recruitment to the mitochondrial membrane by modulating MEK/ERK signal and interacting with Fis1. SIRT5-overexpressing $\mathrm{C}_{2} \mathrm{C}_{12}$ cells contain large and lengthened mitochondria that are uniformly arranged in the cytoplasm partly due to SIRT5-mediated upregulation of Mfn2 and Opa1, whereas SIRT5-silenced cells display small, round mitochondria that are distributed around the nucleus (Polletta et al., 2015). Consistent with these findings, SIRT5mediated fusion promotion in mouse embryonic fibroblasts was also reported by Guedouari, and the results showed that elongated mitochondria and depressed autophagy depended on SIRT5 under starvation conditions (Guedouari et al., 2017). SIRT5 deletion increased the expression of mitochondrial dynamic protein of $51 \mathrm{kDa}$, Fis1, and pDRP1-S637, subsequently causing Drp1 activation and its translocation to mitochondria, thus promoting mitochondrial fission (Guedouari et al., 2017).

The available evidence suggests that Sirt family members located on mitochondria generally promote mitochondrial fusion and/or inhibit mitochondrial fission, and this effect is largely accompanied by a reduction in mitophagy or autophagy. It is hypothesized that fused mitochondria modulated by Sirt subtypes on mitochondria help alleviate stress injury and avoid damage caused by mitophagy.

\section{MORPHOLOGICAL CHARACTERISTICS OF MITOCHONDRIA IN MSCs}

Mitochondria are the major source of energy in the form of ATP, which is produced through oxidative phosphorylation (OXPHOS). The process of OXPHOS and ATP generation occurs in the inner membrane of mitochondria (Gilkerson et al., 2003; Strauss et al., 2008). The shape of mitochondrial cristae and total mitochondria affect electron transport chains and protein complex production, which is significant for bioenergetic output (Beninca et al., 2014; Khacho et al., 2014). On the one hand, mitochondrial morphology is tightly linked to mitochondrial bioenergetics (Hoppins et al., 2007; Cogliati et al., 2013); on the other hand, it is also a reflection of ever-changing dynamics. Generally, well-developed, interlinked mitochondria with complex cristae structures tend to produce energy more efficiently than immature, spherical mitochondria because they have a larger surface area that can hold more intermembrane proteins (Zick et al., 2009). The fused or interconnected morphology of mitochondria is commonly found in metabolically active cells that depend on OXPHOS for energy production (Zhang et al., 2018; Fu et al., 2019). In contrast, cells that utilize glycolytic metabolism for energy production primarily have unfused spherical mitochondria (Seo et al., 2018; Zhang et al., 2018). Such immature mitochondria are metabolically less energetic and less polarized (Collins et al., 2002).

Mitochondrial morphology in embryonic stem cells (ESCs) and iPSCs is generally in an immature state featured by perinuclear-localization and fragmented, spherical, or punctate shapes (Folmes et al., 2011; Zhou et al., 2012). Similar mitochondrial characteristics have been observed in predominantly quiescent non-transplanted hematopoietic stem cells (HSCs) (Papa et al., 2018, 2019; Liang et al., 2020). These immature mitochondria are consistent with the energy state of stem cells, which usually depend on glycolysis as their primary energy source. Although MSCs have glycolysis-dependent 
energy metabolism and immature mitochondria with poorly developed cristae, the shape of their mitochondria is relatively more tubular than that in ESCs and iPSCs (Seo et al., 2018; Fu et al., 2019). ASCs have typical tubular mitochondria that form a robust mitochondrial network (Alicka et al., 2019). Additionally, more than two-thirds of ASCs display small globular and linear tubular structures (Li et al., 2015). Another morphological analysis of mitochondria showed general wiry or tubular mitochondria in BMSCs (Jin et al., 2018). Correspondingly, mitochondria-mediated fission contributes to immature mitochondrial morphology in BMSCs (Feng et al., 2019). These mitochondrial characteristics in MSCs are consistent with the metabolic level of MSCs in which mitochondria maintain low mitochondrial activity, low ROS lever and provide energy primarily through glycolysis (Fillmore et al., 2015; Hu et al., 2018a). However, embryonic mouse neural stem cells (NSCs) have increased mitochondrial lengths and relatively developed networks compared with ESCs or iPSCs, although these cells depend on aerobic glycolytic metabolism (Khacho et al., 2016).

It seems that most quiescent stem cells prefer glycolysis and have immature mitochondrial networks. However, this is a common situation, rather than a fixed and universally applicable criterion. The details referring to mitochondrial morphology and regulatory mechanisms vary among different types of stem cells. One should likely consider other factors beyond energy metabolism to impact mitochondrial morphology and dynamics. Additionally, based on the immature networks dominated by mitochondrial fission, it can be hypothesized that mitochondrial fusion-related factors remain in the low activation state. This does not mean, however, that mitochondrial fusion is not important. In contrast, male germline stem cells with simple and punctate mitochondria are sensitive to a block in fusion, and knockdown of mitofusin or Opal results in dysfunctional mitochondria and dyslipidemia (Senos Demarco et al., 2019). Additionally, deletion of Opa1 or Mfn1/2 destroys the structure of mitochondria and causes cell dysfunction in NSCs (Khacho et al., 2016). These findings suggest that both fission and fusion are indispensable for maintaining a normal mitochondrial morphology in stem cells.
Moreover, even the same types of stem cells undergo metabolic transitions and distinct mitochondrial dynamics due to different pluripotent states and differentiation fates. ESCs have been found to exhibit two stable but epigenetically distinct pluripotent states, named naïve and primed (Zhou et al., 2012; Sperber et al., 2015). Even though naïve ESCs contain under-developed mitochondria, they rely on bivalent metabolism and display a dynamic transition from glycolysis to OXPHOS according to demand (Zhou et al., 2012; Sperber et al., 2015). In contrast, highly glycolytic primed ESCs and epiblast stem cells manifest more mature mitochondria with well-developed cristae compared with naïve ESCs (Zhou et al., 2012; Sperber et al., 2015). With differentiation toward neural progenitor cells, iPSCs gradually form fused mitochondria with well-defined cristae, accompanied by a metabolic switch from glycolysis to OXPHOS (Lorenz et al., 2017). Lymphoid dominant HSCs have longer mitochondria compared with other hematopoietic populations, and Mfn2 is indispensable for HSCs to maintain extensive lymphoid potential (Luchsinger et al., 2016). Even many differentiated cells have more developed mitochondrial networks than stem cells (Cho et al., 2006; Lambertini et al., 2015), and this mitochondrial structure change may not be linear and may suffer complex dynamic changes. Mitochondria display considerable structural diversity in response to different physiological conditions.

Together, mitochondrial networks vary among different types of stem cells, distinct pluripotent states, and specific commitment fates (Table 2). Such variable mitochondrial morphology mediated by mitochondrial fission and fusion is rather sensitive to environment stimulation and is highly plastic.

\section{MITOCHONDRIAL DYNAMICS IN MSC FUNCTION}

Mitochondria undergo specific dynamic changes during stem cell proliferation, migration, differentiation, apoptosis, and aging (Figure 2). However, mitochondrial dynamics regulate cell fate by orchestrating the energy supply, intracellular ROS production and calcium balance.

TABLE 2 | Mitochondrial morphology in stem cells and differentiated cells.

\begin{tabular}{|c|c|c|c|c|}
\hline Stem cell type & Mitochondria morphology & Morphological change & Differentiated fate & References \\
\hline Naïve embryonic stem cells & Rounded to oval mitochondria & \multirow[t]{2}{*}{ Mitochondrial elongation } & \multirow[t]{2}{*}{ Cardiomyocytes } & \multirow{2}{*}{$\begin{array}{l}\text { Zhou et al., 2012; Kasahara et al., 2013; } \\
\text { Sperber et al., 2015; Wang et al., 2017a }\end{array}$} \\
\hline Primed embryonic stem cells & $\begin{array}{l}\text { Elongated mitochondria with } \\
\text { well-defined cristae }\end{array}$ & & & \\
\hline \multirow[t]{2}{*}{ Induced pluripotent stem cells } & \multirow[t]{2}{*}{ Globular mitochondria } & Mitochondrial elongation & Neural progenitor cells & \multirow{2}{*}{$\begin{array}{l}\text { Prieto et al., } 2016 \\
\text { Fang et al., 2016; Lorenz et al., } 2017\end{array}$} \\
\hline & & Mitochondrial elongation & Neurons & \\
\hline \multirow[t]{2}{*}{ Neural stem cells } & \multirow[t]{2}{*}{ Elongated mitochondria } & Mitochondrial fragmentation & Committed progenitors & \multirow{2}{*}{$\begin{array}{l}\text { Khacho et al., 2016; Ribeiro et al., 2019; } \\
\text { Beckervordersandforth et al., } 2017\end{array}$} \\
\hline & & Mitochondrial elongation & Neurons & \\
\hline \multirow[t]{2}{*}{ Mesenchymal stem cells } & \multirow[t]{2}{*}{ Tubular mitochondria } & Mitochondrial elongation & Adipocyte, osteoblasts & \multirow{2}{*}{$\begin{array}{l}\text { Alicka et al., 2019; Feng et al., 2019; Forni } \\
\text { et al., } 2016\end{array}$} \\
\hline & & Mitochondrial fragmentation & Chondrogenic commitment & \\
\hline $\begin{array}{l}\text { Non-transplanted hematopoietic } \\
\text { stem cells }\end{array}$ & $\begin{array}{l}\text { Small and globular } \\
\text { mitochondria }\end{array}$ & Mitochondrial elongation & Lymphoid commitment & \multirow{2}{*}{$\begin{array}{l}\text { Romero-Moya et al., 2013; Papa et al., } \\
\text { 2018; Luchsinger et al., 2016; Liang et al., } \\
2020\end{array}$} \\
\hline $\begin{array}{l}\text { Transplanted hematopoietic } \\
\text { stem cells }\end{array}$ & $\begin{array}{l}\text { Elongated and swollen } \\
\text { mitochondria }\end{array}$ & & & \\
\hline
\end{tabular}




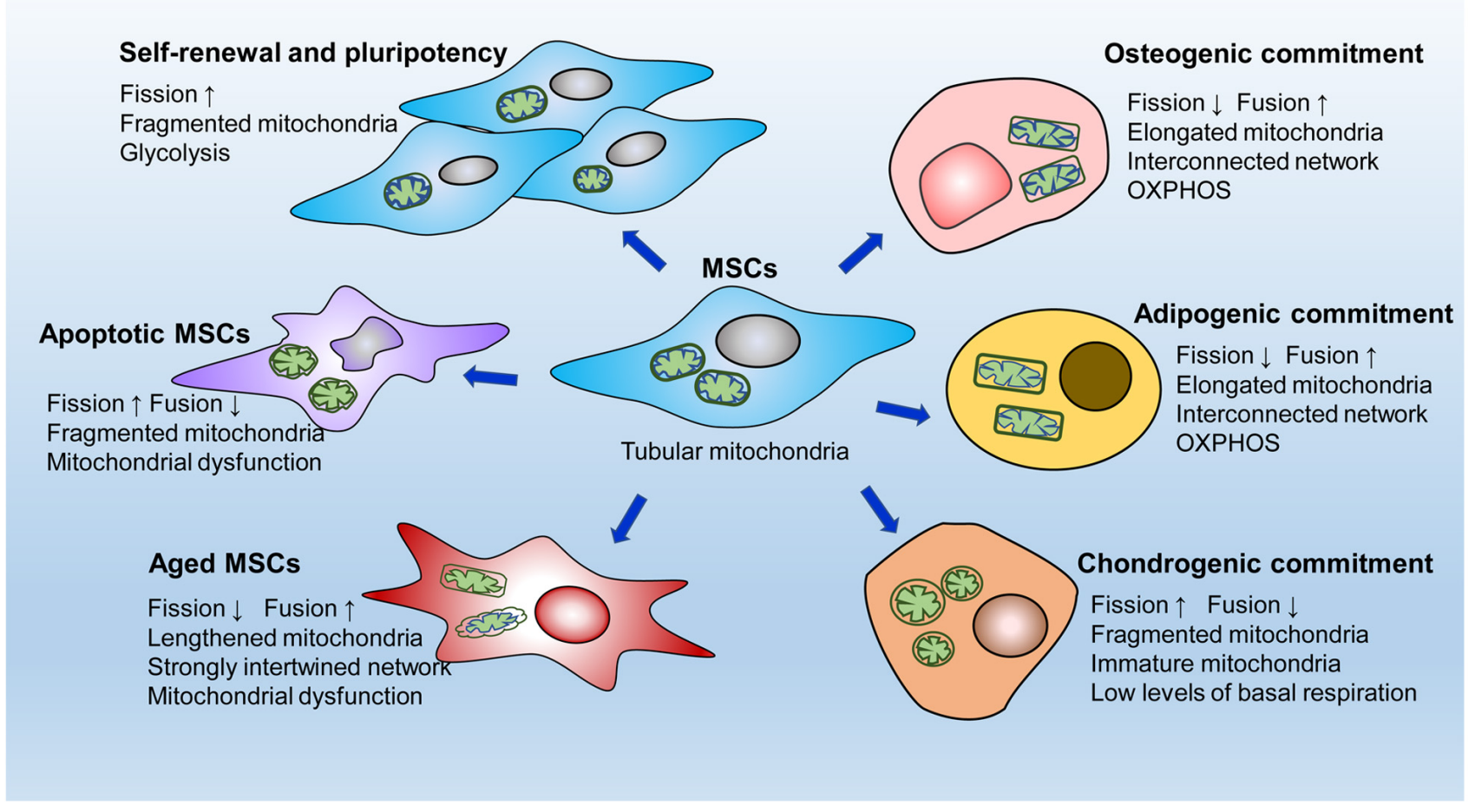

FIGURE 2 | A simple diagram of mitochondrial dynamics in different MSCs behaviors. MSCs contain an immature mitochondrial network characterized by tubular mitochondria. Active mitochondrial fission, adapted to glycolytic dependence on energy production, is critical for the self-renewal and pluripotency of MSCs. After osteogenic or adipogenic induction, MSCs develop elongated mitochondria with interconnected networks. Correspondingly, MSCs undergo dramatic metabolic changes from glycolysis to oxidative phosphorylation for the energy supply. In contrast, in the early stage of chondrogenic commitment, fragmented mitochondria are clearly increased in MSCs accompanied by a low level of basal respiration. Mitochondrial fission is significantly enhanced in apoptotic MSCs, whereas mitochondrial fusion is markedly upregulated in aging MSCs.

\section{Mitochondrial Dynamics in MSC Pluripotency}

As previously mentioned, fragmented mitochondria meet the energy needs of immature MSCs, exerting a fundamental role on intrinsic function (Seo et al., 2018; Zhang et al., 2018). Mitochondrial fission is essential for maintaining stemness in MSCs, and inhibiting fission leads to a reduction in the expression of stemness markers and multidirectional differentiation potential (Feng et al., 2019). During somatic cell reprogramming to iPSCs, cells undergo mitochondrial reconstruction, usually from a mature network toward immature mitochondria, and a metabolic shift, usually from OXPHOS toward glycolysis. Drp1-dependent mitochondrial fission is also necessary for the acquisition of cellular pluripotency during the early stage of embryonic fibroblast induction to form iPSCs (Prieto et al., 2016). Moreover, depletion of fusion-related Mfn1 and Mfn2 activates hypoxia-inducible factor $1 \alpha$ signaling, a necessary mediator of the metabolic switch to glycolysis, thus facilitating the transition in metabolic pattern from OXPHOS to glycolysis (Son et al., 2015). These processes promote the conversion of somatic cells to a pluripotent state (Son et al., 2015). However, over fission induced by Mff overexpression impairs the pluripotency of ESCs and iPSCs, suggesting that active fission should be within a certain threshold to better maintain stem cell function (Zhong et al., 2019). In summary, MSCs prefer mitochondrial fission to mitochondrial fusion to sustain the cell state and function, and active to-fission promotes the self-renewal and pluripotency of stem cells by stimulating glycolysis.

\section{Mitochondrial Dynamics in MSC Differentiation}

In contrast to most stem cells, which have immature mitochondria, stem cells undergoing differentiation usually develop mature or specialized mitochondrial networks. MSCs undergoing differentiation display elongated and interconnected mitochondria accompanied by significantly decreased Drp1 and markedly increased OPA1 expression, indicating that mitochondrial fusion is conducive to MSC differentiation into adipocytes and osteocytes (Feng et al., 2019; Fujiwara et al., 2019). Similarly, Forni et al. induced osteogenesis and adipogenesis in MSCs and demonstrated enhanced mitochondrial biogenesis and network restructuring via mitochondrial fusion mediated by Mfn1 and Mfn2 during the early stages of induction (Forni et al., 2016). Furthermore, Mfn2 knockdown restrains respiratory activity, including basal ATP, maximal respiratory capacity, and $\mathrm{H}^{+}$-leak-related respiratory activity, leading to a loss of differentiation ability (Forni et al., 2016). The fused mitochondrial network facilitates mitochondrial energy 
production through OXPHOS, which corresponds to the energy required in these differentiated cells (Hsu et al., 2016; Li et al., 2017b). Consistently, during the differentiation of human iPSCs into cardiomyocytes, blocking Drp1 induces a metabolic transition from glycolysis toward OXPHOS, resulting in increased differentiation capacity in these cells (Hoque et al., 2018). Neuronal differentiation of iPSCs is closely related to upregulation of Mfn2 expression (Fang et al., 2016). Depletion of Mfn2 induces mitochondrial respiratory dysfunction by inhibiting complexes I and IV enzymatic activity and reducing ATP levels (Fang et al., 2016).

Interestingly, chondrogenesis in MSCs involves a fragmented mitochondrial phenotype at the beginning stage with increased expression of Drp1, Fis1, and Fis2 (Forni et al., 2016), which seems to contradict the finding that mitochondrial fusion is promoted during osteogenic and adipogenic differentiation of MSCs. Cells undergoing chondrogenesis do have low levels of basal respiration at the early commitment phase (Forni et al., 2016). Metabolomic analysis has revealed that catabolic processes occurring during the early stage of chondrogenesis are performed by both glycolysis and mitochondrial respiration (Kwon and Ohmiya, 2013). These metabolic changes differ from the enhanced mitochondrial OXPHOS that occurs during early adipogenic or osteogenic commitment (Chen et al., 2008; Li et al., 2017b), which may partly explain the increased fission to support glycolysis in MSCs during the early stage of chondrogenesis.

Mitochondrial dynamics clearly alter the differentiation fate of MSCs by regulating energy metabolism. However, mitochondrial dynamics-mediated regulation of MSC differentiation is not limited to energy metabolism regulation. Mitochondrial dynamics-dependent mitophagy may also be involved in modulating stem cell differentiation. Evidence has shown that mitochondria become fragmented before mitophagy enhancement (Forni et al., 2016; Marycz et al., 2016), demonstrating that mitochondrial fission is essential for mitophagy or autophagy (Gomes and Scorrano, 2008; Frank et al., 2012), which plays a critical role in MSC differentiation (Marycz et al., 2016; Vidoni et al., 2019). Additionally, although the relationship between mitochondrial dynamics and calcium homeostasis in MSCs has not been revealed, it has been suggested that mitochondrial dynamics can influence the differentiation of stem cells by regulating intracellular calcium balance. Zhong et al. (2019) reported that excess mitochondrial fission exacerbated cytosolic $\mathrm{Ca}^{2+}$ entry and CaMKII activity, resulting in the degradation of $\beta$-catenin and ultimately impairing the differentiation and embryonic development of iPSCs. Mfn2 negatively regulates calcineurin/NFAT activity by intracellular $\mathrm{Ca}^{2+}$ buffering, thereby maintaining HSCs with extensive lymphoid potential (Luchsinger et al., 2016). Kasahara et al. (2013) demonstrated that gene trapping of Mfn2 or OPA1 was sufficient to inhibit the differentiation of ESCs into cardiomyocytes. Mechanically, mitochondrial fusion orchestrates $\mathrm{Ca}^{2+}$ and calcineurin A to further affect Notch1-mediated suppression of the cardiomyocyte transition (Kasahara et al., 2013).

In short, although mitochondrial fission and mitochondrial fusion may exert pleiotropic effects during MSC differentiation depending on specific lineage commitment and different stages, we hypothesize that such mitochondrial dynamics undergo specific transformations to ensure the ever-changing energy demands and calcium balance, which is fundamental for the multidirectional differentiation of MSCs.

\section{Mitochondrial Dynamics in MSC Senescence}

Long-term in vitro amplification is a common way that can induce senescence of MSCs (Kasper et al., 2009). After a certain number of cell divisions (7-12 passages), senescent cells increase, which is characterized by morphological abnormalities, enlargement, and increase of senescenceassociated $\beta$-galactosidase positive cells. The long-term MSCs cultures (more than 100 passages) derived from rat have been found to exhibit increased susceptibility to senescence and have non-tumorigenic (Wagner et al., 2008; Geissler et al., 2012). Karyotype analysis in BMSCs reveals that aneuploidy chromosomal alterations may occurs during population doublings, but they became senescent without transformation features (Tarte et al., 2010).

Lengthened mitochondria often occur in various aging cells (Mai et al., 2010; Lin et al., 2015). Aged MSCs also exhibit a strong and complicated interconnected network that is distributed evenly in the cytoplasm, suggesting a potentiation of fusion processes (Geissler et al., 2012). p-Drp1 expression has been reported to be greatly downregulated, whereas Mfn2 expression is markedly upregulated in passage 12 (P12) BMSCs compared with those in P4 BMSCs, suggesting that these cells undergo aging accompanied by mitochondrial fusion (Li et al., 2019). Consistent with these observations, P7 ASCs have large tubular mitochondria forming an intertwined network that is regulated by Mfn1, Opa1, and Fis1 (Stab et al., 2016). In contrast, P2 ASCs show small tubular mitochondria forming a slightly interconnected network (Stab et al., 2016). Excessive mitochondrial fusion may adversely affect cells by altering ROS levels. Prolonged or giant mitochondria have been reported to augment ROS generation and weaken mitochondrial respiration activity in deferoxamine-induced senescent cells (Yoon et al., 2006). Furthermore, blocking mitochondrial fission, by overexpression of Drp1-K38A (active site is mutated in Drp1) and Fis1- $\Delta \mathrm{TM}$ (transmembrane domain is deleted in Fis1), successfully leads to a senescent phenotype with ROS elevation in normal cells (Yoon et al., 2006). Additionally, the reduction in Drp1 levels during vascular aging exacerbates endothelial cell dysfunction by increasing mitochondrial ROS and suppressing autophagic flux, while the antioxidant $\mathrm{N}$-acetyl-cysteine restores autophagosome clearance and improves angiogenesis in senescent endothelial cells (Lin et al., 2015).

Notably, increased mitochondrial fusion during aging is not always harmful (Stab et al., 2016). During fusion, depolarized mitochondria and normal mitochondria can join together to exchange their contents, further aiding in damaged mitochondrial repair and membrane potential maintenance (Twig et al., 2008b; Meyer et al., 2017). Of course, excessive fusion 
can be detrimental when depolarized and damaged mitochondria are overloaded, which is conducive to mitochondrial dysfunction during aging (Ikeda et al., 2015; Lang et al., 2017).

\section{Mitochondrial Dynamics in MSC Apoptosis}

Conversely, cell apoptosis is usually accompanied by abnormal rupture of mitochondria in MSCs. Apoptotic ASCs isolated from equine metabolic syndrome (EMS) horses contain notably increased fragmented mitochondria (Kornicka et al., 2019). Analogously, prominently increased expression of Fis1 and decreased Mfn1 and Mfn2 expression have been reported in apoptotic human umbilical cord MSCs induced by monocrotophos exposure (Srivastava et al., 2018). Ma et al. (2019) demonstrated that dexamethasone increased mitochondrial fission and meanwhile diminished mitochondrial fusion. Mitochondrial fission was promoted by increased Fis1 and Mff expression, whereas the mitochondrial fusion was inhibited by decreased Mfn1 and Mfn2 expression. These mitochondrial dynamics alterations contributes to apoptosis enhancement and osteogenic suppression of BMSCs (Ma et al., 2019). Correspondingly, treatment with mdivi-1, an inhibitor of Drp1, dramatically ameliorated hydrogen peroxide $\left(\mathrm{H}_{2} \mathrm{O}_{2}\right)$ induced cellular apoptosis and death in human periodontal ligament stem cells (PDLSCs) and human $\mathrm{W}_{8} \mathrm{~B}_{2}+$ cardiac cells (Rosdah et al., 2017; He et al., 2018). Moreover, glucose/serumdeprived/hypoxia treatment-induced apoptosis in MSCs is reduced by increased glycolytic efficacy, which is modulated by the leptin/OPA1/SGLT1 signaling pathway (Yang et al., 2019).

The role of mitochondrial fission in modulating stem cell apoptosis is still unclear. Mitochondrial outer membrane permeabilization (MOMP) mediates the cascade conduction of many apoptotic signals. Arnoult et al. (2005) observed that $\mathrm{Bax} / \mathrm{Bak}$ could facilitate the release of deafness dystonia protein 1 homolog, a member of mitochondrial intermembrane chaperone, into the cytoplasm, promoting its binding to the C-terminus of Drp1, this further results in Drp1 recruitment to mitochondria and Drp1-dependent mitochondrial fission. This Drp1-mediated mitochondrial fragmentation is vital for mitosis, which is involved in caspase-independent cell death (Arnoult et al., 2005). Earlier findings suggested that overexpression of Mfn1 reduces apoptotic HeLa cells induced by etoposide by impeding Bax transport to mitochondria and cytochrome c release (Sugioka et al., 2004). Intriguingly, other reports have shown that Drp1mediated mitochondrial fission prevents cell apoptosis, especially $\mathrm{Ca}^{2+}$-related death (Szabadkai et al., 2004; Jahani-Asl and Slack, 2007). Specific upregulation of Drp1 leads to division of the mitochondrial network and damages the connectivity of the mitochondrial lumen, separating mitochondria from the endoplasmic reticulum, the source of calcium, which decreases $\mathrm{Ca}^{2+}$ absorption and ultimately abrogates $\mathrm{Ca}^{2+}$ overloadinduced apoptosis (Szabadkai et al., 2004). However, these mitochondrial fission-dependent antiapoptotic events have not been demonstrated in stem cells.

Although current studies have shown that the apoptotic process of stem cells is dominated by mitochondrial fission, different apoptosis-inducing factors should be further studied to comprehensively understand the effect of mitochondrial dynamics on apoptosis. Overall, the interaction between mitochondrial dynamics and apoptotic signals is complicated. In response to different apoptotic or death pathway stimuli, mitochondrial dynamics may either support or restrain apoptosis. Increased apoptosis is usually attributed to enhanced mitochondrial fission since these processes act on Bcl-2 family dependent apoptotic pathways and related molecules. The effectiveness and mechanisms involved in mitochondrial dynamic-mediated alleviation of cell death caused by $\mathrm{Ca}^{2+}$ overload requires further confirmation.

\section{MITOCHONDRIAL DYNAMICS IN MSCs UNDER STRESS}

Mitochondria can sense many stresses and influence cell survival and function by regulating a variety of signaling molecules. Mitochondrial dynamics play an important role in the mitochondrial stress response (Youle and van der Bliek, 2012; Meyer et al., 2017; Eisner et al., 2018). At present, the stress response of mitochondrial dynamics in MSCs primarily includes oxidative stress, metabolic stress, and some exogenous stimuli, such as physical stress and toxins.

\section{Mitochondrial Dynamics in MSCs Under Oxidative Stress}

Oxidative stress occurs when the balance of the oxidative stress and antioxidant systems breaks down, which subsequently causes cellular damage. During physiological or repair processes, stem cells inevitably suffer attacks caused by oxidative stress. In fact, oxidative stress is a well-explored mechanism in regulating stem cell fate (Ko et al., 2012; Tan and Suda, 2018). Overgeneration of ROS indicates the occurrence of oxidative stress in tissues (Tan and Suda, 2018; Vina et al., 2020). Under pathological conditions, excessive ROS can be produced by mitochondria, which in turn leads to the inactivation of mitochondrial components (Zorov et al., 2014). The damaged members involved in mitochondrial dynamics may destroy the mitochondrial morphology and structure, resulting in a vicious cycle of continuous ROS release (Ježek et al., 2018). This reflects the complex interaction between mitochondrial dynamics and ROS generation.

Oxidative stress with ROS evaluation induced directly by $\mathrm{H}_{2} \mathrm{O}_{2}$ treatment leads to mitochondrial fragmentation in hMSCs; moreover, the combination of $N$-acetylcysteine, a biologic antioxidant, and ascorbic acid 2-phosphate, an oxidation-resistant derivative of ascorbic acid, successfully inhibits mitochondrial fission, decreases ROS production, and stabilizes mitochondrial membrane potential (Li et al., 2015). In another similar study of oxidative stress conditions stimulated by serum deprivation and hypoxia treatment, BMSCs had increased mitochondrial fragmentation along with upregulation of p-Drp1 Ser616 expression and downregulation of Mfn2 expression (Deng et al., 2020). In addition, an in vitro study underscored that $\mathrm{CoCl}_{2}$, a hypoxia mimetic, promoted mitochondrial fission in PDLSCs mediated by Drp1 elevation (He et al., 2018). Targeted 
inhibition of Drp1 markedly increased ATP levels, suppressed ROS generation, and eventually reduced cell apoptosis, indicating the important role of the ROS-Drp1-dependent mitochondrial pathway in $\mathrm{CoCl}_{2}$-induced apoptosis in PDLSCs (He et al., 2018). These findings suggest that high ROS levels and oxidative stress generally lead to abnormal mitochondrial dynamics, especially excessive mitochondrial fission. Reducing ROS levels helps to restore normal mitochondrial dynamics. Moreover, the regulation of mitochondrial dynamics can also be beneficial for reversing ROS overgeneration.

Unlike the high level of ROS, which is always associated with cell damage and disease, low or normal ROS level has been shown to have a positive effect on cell homeostasis and function via participating in signal transduction and promoting mitophagy (Shadel and Horvath, 2015; Palmeira et al., 2019). Early outbreaks of transient oxidative phosphorylation and elevated ROS in somatic cells promote NRF2 transcription factor activity, which further initiates the hypoxia inducible factor $\alpha$-mediated glycolytic shift in early reprogramming (Hawkins et al., 2016). During reprogramming toward iPSCs, mitochondria undergo reconstruction dominated by enhanced mitochondrial fission, gradually forming an immature state instead of a mature mitochondrial network (Vazquez-Martin et al., 2012; Prieto et al., 2016; Lisowski et al., 2018). Compared with somatic cells, stem cells including MSCs have low ROS levels and immature mitochondrial networks (Hsu et al., 2016; Lisowski et al., 2018). Therefore, it is speculated that the changes in mitochondrial dynamics associated with low ROS levels are conducive to mitochondrial remodeling and adaptive changes.

\section{Mitochondrial Dynamics in MSCs Under Metabolic Stress}

Studies on the effects of metabolic stress on mitochondrial dynamics mainly involve abnormalities in glucose and lipid metabolism. High levels of fatty acids alone or in combination with high glucose induce an increase in mitochondrial fragmentation (Molina et al., 2009). Dysfunctional ASCs isolated from patients with type 2 diabetes exhibit a similar trend in mitochondrial phenotype, in which overexpression of Fis 1 causes fragmented, round mitochondria, and mitochondrial autophagy is also impaired, as indicated by reduced parkin RBR E3 ubiquitin protein ligase (PRKN, better known as Parkin) expression (Alicka et al., 2019). The Parkin is a crucial mitophagy regulator and its activation can build ubiquitin chains to ubiquitinating outer membrane protein on damaged mitochondria to label them for degradation in lysosomes (Bingol and Sheng, 2016). These findings are consistent with previous research by the same team, which showed that increased ASC apoptosis derived from EMS horses resulted in enhanced mitochondrial fission compared with the healthy control (Marycz et al., 2018). Similarly, metabolic syndrome impairs the swine ASC mitochondrial structure featured by an increase in mitochondrial fission and reduction of mitochondrial fusion (Farahani et al., 2020). Yu et al. (2006) have found that hyperglycemia-induced mitochondrial fission promotes ROS production and its periodic fluctuations, and either fission inhibition by Drp1 inhibition or fusion induction by Mfn2 overexpression prevents an increase in ROS induced by hyperglycemia.

\section{Mitochondrial Dynamics in MSCs Under Physical Stress and Toxins}

Limited studies have indicated the potential impacts of physical stimuli or exogenous toxins on the mitochondrial dynamics of MSCs. Patten et al. (2019) observed that mitochondrial length in human BMSCs was slightly increased $4 \mathrm{~h}$ after exposure to 2 Gy radiation. They further demonstrated that Opal knockdown in mouse embryonic fibroblasts induced a decline in their adaptation to radiation, suggesting that mitochondrial networks may also be involved in the regulation of BMSC adaptation to radiation (Patten et al., 2019). Yin et al. (2017) revealed that low-level laser exposure facilitated mitochondrial biogenesis via upregulation of molecules associated with both mitochondrial fusion (Mfn1, Mfn2, and Opa-1) and mitochondrial fission (Fis1, Drp1, and MTP18), which contribute to elevated proliferation of BMSCs. In an in vitro study, methamphetamine exposure dampened the osteogenic differentiation of BMSCs due to abnormal OXPHOS and reduced ATP generation and mitochondrial membrane depolarization. These mitochondrial malfunctions were attributed to damaged mitochondrial biogenesis and mitochondrial fusion (Shen et al., 2018a). Similarly, treatment with carbon black Printex 90, a representative carbonaceous particle toxicant, induced mitochondrial dysfunction in BMSCs, which is closely related to suppressed mitochondrial biogenesis and mitochondrial dynamics, ultimately resulting in impaired osteogenic potential of BMSCs (Shen et al., 2018b).

\section{Multifaceted Effects of Mitochondrial Dynamics Under Stress}

According to the literature, stress often results in fragmentation but not elongation events in mitochondria in MSCs, which is attributed to dramatic enhanced mitochondrial fission with/without inhibited mitochondrial fusion (He et al., 2018; Marycz et al., 2018; Shen et al., 2018a; Ma et al., 2019). In addition, the suppression effect on both mitochondrial fission and fusion was observed in BMSCs when they were treated with high levels of ferric ammonium citrate, a commonly used agent to induce iron overload (Yao et al., 2019). In contrast, Marycz et al. (2019) suggested that ASCs derived from metabolic syndrome horses displayed abnormal dysregulated and mixed mitochondria due to hyperactive mitochondrial fission and fusion. Moreover, stress-induced mitochondrial hyperfusion has been proposed by Tondera et al. (2009) as a particular prosurvival response to stress, and their research confirmed that exposure to a low dose of UV irradiation or actinomycin $\mathrm{D}$ efficiently induces hyperfusion of mitochondria in mouse embryonic fibroblasts within $9 \mathrm{~h}$. Similarly, mouse embryonic fibroblasts show elongated mitochondria after starvation treatment, which protects them from autophagy-induced mitochondrial clearance (Gomes et al., 2011). Jendrach et al. (2008) observed that short-term and low doses of $\mathrm{H}_{2} \mathrm{O}_{2}$ result 
in a transitory increase in mitochondrial fusion in human umbilical vein endothelial cells. This enhanced mitochondrial fusion is beneficial to damaged mitochondria, in which mitochondrial DNA and membrane potential are sustained and the energy supply capacity is maximized to enhance adaptation under stress (Tondera et al., 2009; Gomes et al., 2011; Meyer et al., 2017).

Therefore, it is reasonable to conclude that different stressors and stress intensities can lead to complex changes in mitochondrial dynamics in MSCs, which are involved in fission and/or fusion activity. Mitochondrial dynamics seem to be central to bridging the gap between external stress and MSC function (Figure 3). Fusion events may act as a compensatory reaction in response to weak stress to better promote survival. In many cases, mitochondrial elongation is beneficial for resisting stress (Agarwal et al., 2016; Yang et al., 2019). However, upregulated fusion does not always mean elevated function. For example, senescent cells usually have a highly fused mitochondrial network, but their functions are relatively poor (Lin et al., 2015; D'Amico et al., 2019). Likewise, mitochondrial fragmentation is not always the result of maladaptation. Under stress states, impaired mitochondria may cause damage by ROS or other overgenerated harmful byproducts and abnormal accumulation of $\mathrm{Ca}^{2+}$ (Shaughnessy et al., 2014; Park et al., 2017; Dilberger et al., 2019). Accordingly, timely and proper clearance of damaged mitochondria is imperative to cell survival and function. Mitophagy is the main way to selectively eliminate damaged or old mitochondria (Twig et al., 2008a; Ashrafi and Schwarz, 2013). The suppression of mitophagy impairs cell adaptability to stress and has been implicated in diabetes and neurodegenerative and cardiovascular aging diseases (Wallace, 2005; Egan et al., 2011; Marycz et al., 2016; Baechler et al., 2019). Mitochondria rely on mitochondrial fission to produce different daughter units. The daughter units with healthier membrane potentials will continue to participate in the dynamic cycle and facilitate recovery, whereas depolarizing mitochondria can be removed by mitophagy (Twig et al., 2008a). Inhibition of mitochondrial fission impairs mitophagy, while transitory enhanced mitochondrial fission acts as a protective strategy via cooperation with mitophagy (Twig et al., 2008a; Youle and van der Bliek, 2012). Stress most often induces hyperfission or abnormal mitochondrial dynamics, thus stimulating or worsening cell damage or death. Taken together, these findings suggest that the coordination of mitochondrial fission and mitochondrial fusion is a pivotal cytoprotective mechanism for cellular renovation and homeostasis under stress.

\section{MSC-BASED MITOCHONDRIAL THERAPEUTICS}

The coordination of mitochondrial fission and mitochondrial fusion is essential for maintaining integrity and function

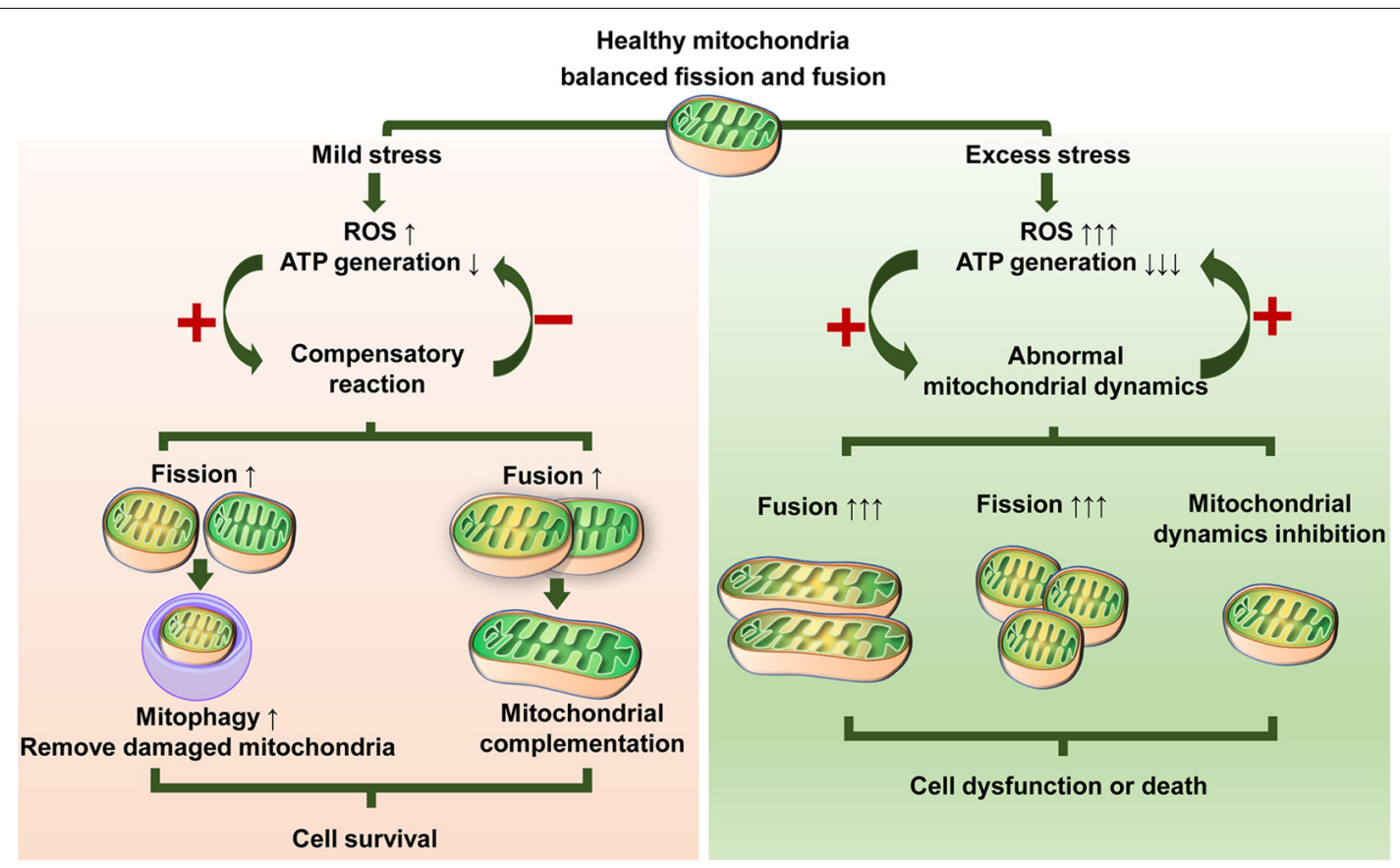

FIGURE 3 | Illustration showing possible responses of mitochondrial dynamics to stress. Different stressors and stress levels lead to altered mitochondrial fission or/and fusion. Mild stress induces moderate ROS production and decreased ATP generation, which triggers adaptive changes in mitochondrial dynamics. Mitochondria can maintain their quality either by enhanced mitochondrial fission to remove damaged mitochondria or enhanced mitochondrial fusion to share components that, in turn, can relieve oxidative stress and fortify the energy supply, thus promoting survival. Excess stress causes a dramatic ROS increase and ATP exhaustion, which contributes to abnormalities in mitochondrial dynamics. In turn, abnormal mitochondrial dynamics exacerbate this situation via a vicious circle of continuous ROS elevation and/or ATP reduction, therefore inducing or worsening cell dysfunction or death. 
of MSCs. Some crucial proteins that control mitochondrial dynamics play particularly important roles in regulating MSC fates. Based on these regulators, many strategies are being developed to guide mitochondrial dynamics to govern cell fate, thus improving the efficacy of MSC-based tissue repair.

Mdivi-1 is widely used as a selective Drp1 inhibitor that can inhibit the GTPase activity of Drp1. Some researchers have demonstrated the protective effect of mdivi-1 against apoptosis in stem cells under pathological or stress conditions. Targeted inhibition of Drp1 with mdivi-1 increases mitochondrial fission and protects human PDLSCs against $\mathrm{H}_{2} \mathrm{O}_{2}$-induced cell dysfunction (He et al., 2018). Mdivi-1 treatment rescues the EMS-induced abnormal mitochondrial network in ASCs, which subsequently reverses the senescence of ASCs, as indicated by suppression of p53, p21, and p62 expression (Kornicka et al., 2019). Moreover, short-term or intermittent administration of mdivi-1 is more beneficial for resisting tissue damage (Jheng et al., 2015). Single administration of mdivi-1 reverses the excessive fission of mitochondria and diminishes myocardial ischemia/reperfusion-induced damage in diabetic mice (Ding et al., 2017). Intermittent application of mdivi-1 1 and $16 \mathrm{~h}$ before insulin stimulation can improve insulin resistance in the skeletal muscles of obese mice (Jheng et al., 2012). Other mitochondrial fission inhibitors include P110, which blocks Drp1/Fis1 interactions, and dynasore, which non-selectively dampens GTPase activity. Several studies have reported that these inhibitors can prevent ischemia/reperfusion-induced damage in mouse hearts, suggesting their potential application for stem cells under oxidative stress conditions (Disatnik et al., 2013; Gao et al., 2013).
Gene modification and epigenetic regulation are also used to regulate mitochondrial dynamics and render MSCs more favorable for bone tissue repair. Silencing Mfn2 can diminish ROS levels and reverse the aging phenotype induced by deletion of the FGF21 in BMSCs (Li et al., 2019). Pretreatment of BMSCs with miR181-c activates the AMPK-Mfn1 signaling pathways, consequently reversing $\mathrm{H}_{2} \mathrm{O}_{2}$-induced negative effects on proliferation, migration, and paracrine activity in BMSCs (Fan et al., 2019). Mfn1 silencing abrogates the protective effect of miR-181c on BMSCs under oxidative stress conditions (Fan et al., 2019). Inhibiting miR-155-5p or Mfn2-siRNA reverses mitochondrial hyperfusion-mediated senescence in MSCs, and transplantation of aged MSCs pretreated with anti-miR-155-5p significantly ameliorates cardiac dysfunction in an infarction mouse model (Hong et al., 2020). miR-214 improves fibroblast differentiation of ASCs by directly binding to the Mfn2 $3^{\prime}$ UTR, enhancing ASC-mediated repair in pelvic floor dysfunction in rats with birth trauma. Injection of a miR-214 inhibitor or overexpression of Mfn2 can counteract the therapeutic effect of ASCs in rat urinary tissues (Wu et al., 2017). Local application of lgr5-overexpression MSCs at the fracture site is reported as a superior method in augmenting bone healing in mice because of the positive regulation of mitochondrial dynamics and Wnt/ERK signaling pathways (Lin et al., 2019).

In addition to the targeted strategies described above, some compounds can also improve the biological activities of MSCs by modulating mitochondrial dynamics (Table 3). These studies suggest a complex regulatory network induced by mitochondrial dynamics that affects different MSC behaviors. Given the extensive and complicated role of mitochondrial dynamics, it is

TABLE 3 | A summary of compounds for mitochondrial dynamics modulation in MSCs.

\begin{tabular}{|c|c|c|c|c|}
\hline Compounds & $\begin{array}{l}\text { MSC } \\
\text { types }\end{array}$ & $\begin{array}{l}\text { Mitochondrial dynamics } \\
\text { effect and mechanism }\end{array}$ & Effect on MSCs & References \\
\hline Fibroblast growth factor 21 & $\begin{array}{l}\text { Human } \\
\text { BMSCs }\end{array}$ & 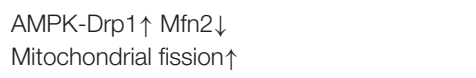 & Senescence $\downarrow$ & Li et al., 2019 \\
\hline $\begin{array}{l}\text { N-Acetylcysteine and Ascorbic } \\
\text { Acid } \\
\text { 2-Phosphate }\end{array}$ & $\begin{array}{l}\text { Human } \\
\text { ASCs }\end{array}$ & $\begin{array}{l}\text { Drp1 S616 translocation } \downarrow \\
\text { Mitochondrial fission } \downarrow\end{array}$ & $\begin{array}{l}\text { Mitochondrial function } \uparrow \\
\text { mitoptosis } \downarrow\end{array}$ & $\begin{array}{l}\text { Li et al., } 2015 \\
\text { Li et al., } 2017 \text { a }\end{array}$ \\
\hline Leptin & $\begin{array}{l}\text { Human } \\
\text { BMSCs }\end{array}$ & $\begin{array}{l}\text { Opa1-mediated } \\
\text { mitochondrial fusion } \uparrow\end{array}$ & $\begin{array}{l}\text { Survival in hypoxia } \uparrow \\
\text { Glucose/serum-deprived/hypoxia-induced } \\
\text { apoptosis } \downarrow\end{array}$ & $\begin{array}{l}\text { Yang et al., } 2019 \\
\text { Yang et al., } 2018\end{array}$ \\
\hline Haemin & $\begin{array}{l}\text { Human } \\
\text { BMSCs }\end{array}$ & $\begin{array}{l}\text { p-Drp1 ser616 } \downarrow \text { Mfn2 } \uparrow \\
\text { Mitochondrial fission } \downarrow\end{array}$ & $\begin{array}{l}\text { SD/H-induced apoptosis } \downarrow \\
\text { Myocardial infarction-induced damage in mice } \downarrow\end{array}$ & Deng et al., 2020 \\
\hline Succinate & $\begin{array}{l}\text { Human } \\
\text { MSCs }\end{array}$ & $\begin{array}{l}\text { MAPK-P38p-Drp-1个 } \\
\text { mitochondrial fission } \uparrow\end{array}$ & $\begin{array}{l}\text { Migration } \uparrow \\
\text { mice skin wound healing } \uparrow\end{array}$ & Ko et al., 2017 \\
\hline $\begin{array}{l}\text { Pyruvate kinase muscle } \\
\text { isoenzyme } 2\end{array}$ & $\begin{array}{l}\text { Rat } \\
\text { BMSCs }\end{array}$ & $\begin{array}{l}\text { Drp } 1 \uparrow \text { Fis } 1 \uparrow \text { Mff } \uparrow \\
\text { Opa1 } \downarrow \text { Mfn2 } 2 \\
\text { mitochondrial fission } \uparrow\end{array}$ & $\begin{array}{l}\text { Osteogenic commitment } \downarrow \\
\text { Adipogenic commitment } \uparrow\end{array}$ & Guo et al., 2020 \\
\hline Melatonin & $\begin{array}{l}\text { Mouse } \\
\text { BMSCs }\end{array}$ & $\begin{array}{l}\text { Opa1 } \downarrow \text { Mfn } 1 \downarrow \\
\text { mitochondrial fusion } \downarrow\end{array}$ & $\begin{array}{l}\text { Chronic kidney disease-related cellular } \\
\text { senescence }\end{array}$ & Han et al., 2019 \\
\hline
\end{tabular}


necessary to be more precise and prudent in exploring targeted regulatory strategies.

\section{CONCLUSION AND PERSPECTIVES}

Mitochondrial dynamics are closely related to the fate determination of MSCs. The coordinated collaboration of mitochondrial fission and mitochondrial fusion is of great significance in the self-renewal, multilineage differentiation, and stress response of MSCs. Proper regulation of mitochondrial dynamics benefits MSCs in sustaining viability, promoting differentiation, and resisting apoptosis, aging, and stress damage. Since mitochondrial dynamics vary with different MSC commitments and ways to participate in the response, and mediation of mitochondrial dynamics should be investigated in diverse MSC differentiation populations. In addition, the mechanism of mitochondrial dynamics in regulating the fate of stem cells includes energy metabolism alteration, oxidative stress modulation, and calcium homeostasis regulation. However, how these regulatory pathways mediate the interaction between mitochondrial dynamics and MSC behaviors requires further elucidation. Additionally, mitochondrial morphology and quality are integrally connected via mitochondrial biogenesis, fission, fusion, and mitophagy, and their crosstalk in MSC fates merits further exploration.

Stimuli such as oxidative stress often induce single changes in mitochondrial dynamics, especially fission activation, in which a strategy unilaterally targeting mitochondrial fission can achieve desirable results with reduced risk. However, the situation is sometimes complicated, and differentiation suppression in MSCs is accompanied by both abnormal fission and fusion (Shen et al., 2018b; Marycz et al., 2019; Yao et al., 2019). In such cases, changes in mitochondrial dynamics may involve a variety of dynamic-related proteins. Therefore, strategies targeting the mediation of mitochondrial dynamics will be complicated and risky and should therefore be carefully evaluated. It is also noteworthy that increased mitochondrial fragmentation

\section{REFERENCES}

Abdul-Rahman, O., Kristóf, E., Doan-Xuan, Q. M., Vida, A., Nagy, L., Horváth, A., et al. (2016). AMP-Activated Kinase (AMPK) activation by AICAR in human white adipocytes derived from pericardial white adipose tissue stem cells induces a partial beige-like phenotype. PLoS One 11:e0157644. doi: 10.1371/ journal.pone.0157644

Agarwal, S., Yadav, A., Tiwari, S. K., Seth, B., Chauhan, L. K., Khare, P., et al. (2016). Dynamin-related protein 1 inhibition mitigates bisphenol A-mediated alterations in mitochondrial dynamics and neural stem cell proliferation and differentiation. J. Biol. Chem. 291, 15923-15939. doi: 10.1074/jbc.M115.709493

Alicka, M., Major, P., Wysocki, M., and Marycz, K. (2019). Adipose-Derived mesenchymal stem cells isolated from patients with Type 2 diabetes show reduced "Stemness" through an altered secretome profile, impaired antioxidative protection, and mitochondrial dynamics deterioration. J. Clin. Med. 8:765. doi: $10.3390 / \mathrm{jcm} 8060765$

Arnoult, D., Rismanchi, N., Grodet, A., Roberts, R. G., Seeburg, D. P., Estaquier, J., et al. (2005). Bax/Bak-dependent release of DDP/TIMM8a promotes Drp1mediated mitochondrial fission and mitoptosis during programmed cell death. Curr. Biol. 15, 2112-2118. doi: 10.1016/j.cub.2005.10.041 may be due to enhanced fission and/or suppressed fusion, while elongated mitochondria are attributed to inhibited fission and/or intensified fusion. An accurate and comprehensive assessment is necessary to obtain the simplest but most effective strategy.

Although still at an early stage, modulating mitochondrial dynamics by various means to guide MSCs to better promote tissue repair is an emerging regulatory strategy that shows great potential in the future of regenerative medicine. More importantly, the definite crosstalk between mitochondrial dynamics and MSC fate should be clarified in detail before any conclusions can be drawn regarding how to direct mitochondrial fission or mitochondrial fusion toward controlling MSC behaviors.

\section{AUTHOR CONTRIBUTIONS}

LR was responsible for conceptualizing this review and writing the original draft. XDC was involved in the conceptualization, funding acquisition, and review and editing of the manuscript. $\mathrm{XBC}$ and JL participated in provision of resources and editing figures. BC contributed to funding acquisition, and participated in supervision, editing and revision of the manuscript. JX contributed to the editing, improving and finalization of the manuscript. All authors approved the final version of the manuscript and agreed to be accountable for this work.

\section{FUNDING}

We thank the financial support from National Natural Science Foundation of China (Grant No. 81900976), Guangdong Basic and Applied Basic Research Foundation (Grant No. 2020A151501025), Guangzhou Foundation for Science and Technology Planning Project, China (Grant No. 201704020063), and Fundamental Research Funds for the Central Universities (Grant No. 19ykpy80).

Ashrafi, G., and Schwarz, T. L. (2013). The pathways of mitophagy for quality control and clearance of mitochondria. Cell Death Diff. 20, 31-42. doi: 10.1038/ cdd.2012.81

Baechler, B. L., Bloemberg, D., and Quadrilatero, J. (2019). Mitophagy regulates mitochondrial network signaling, oxidative stress, and apoptosis during myoblast differentiation. Autophagy 15, 1606-1619. doi: 10.1080/15548627. 2019.1591672

Beckervordersandforth, R., Ebert, B., Schaffner, I., Moss, J., Fiebig, C., Shin, J., et al. (2017). Role of mitochondrial metabolism in the control of early lineage progression and aging phenotypes in adult hippocampal neurogenesis. Neuron 93, 560-573.e6. doi: 10.1016/j.neuron.2016.12.017

Beninca, C., Planaguma, J., de Freitas Shuck, A., Acin-Perez, R., Munoz, J. P., de Almeida, M. M., et al. (2014). A new non-canonical pathway of Galpha(q) protein regulating mitochondrial dynamics and bioenergetics. Cell Signal 26, 1135-1146. doi: 10.1016/j.cellsig.2014.01.009

Bereiter-Hahn, J., and Vöth, M. (1994). Dynamics of mitochondria in living cells: shape changes, dislocations, fusion, and fission of mitochondria. Microsc. Res. Tech. 27, 198-219. doi: 10.1002/jemt.1070270303

Bianco, P. (2014). "Mesenchymal" stem cells. Annu. Rev. Cell Dev. Biol. 30, 677-704. doi: 10.1146/annurev-cellbio-100913-113132 
Bingol, B., and Sheng, M. (2016). Mechanisms of mitophagy: PINK1, Parkin, USP30 and beyond. Free Radical. Biol. Med. 100, 210-222. doi: 10.1016/j. freeradbiomed.2016.04.015

Carling, D. (2004). The AMP-activated protein kinase cascade-a unifying system for energy control. Trends Biochem. Sci. 29, 18-24. doi: 10.1016/j.tibs.2003. 11.005

Chakraborty, P. K., Murphy, B., Mustafi, S. B., Dey, A., Xiong, X., Rao, G., et al. (2018). Cystathionine $\beta$-synthase regulates mitochondrial morphogenesis in ovarian cancer. FASEB J. 32, 4145-4157. doi: 10.1096/fj.201701095R

Chamberlain, G., Fox, J., Ashton, B., and Middleton, J. (2007). Concise review: mesenchymal stem cells: their phenotype, differentiation capacity, immunological features, and potential for homing. Stem Cells 25, 2739-2749. doi: 10.1634/stemcells.2007-2197

Chang, C. R., and Blackstone, C. (2007). Cyclic AMP-dependent protein kinase phosphorylation of Drp1 regulates its GTPase activity and mitochondrial morphology. J. Biol. Chem. 282, 21583-21587. doi: 10.1074/jbc.C70008 3200

Chen, C. T., Shih, Y. R., Kuo, T. K., Lee, O. K., and Wei, Y. H. (2008). Coordinated changes of mitochondrial biogenesis and antioxidant enzymes during osteogenic differentiation of human mesenchymal stem cells. Stem Cells 26, 960-968. doi: 10.1634/stemcells.2007-2509

Chen, H., and Chan, D. C. (2005). Emerging functions of mammalian mitochondrial fusion and fission. Hum. Mol. Genet. 14, R283-R289. doi: 10. 1093/hmg/ddi270

Chen, Q., Shou, P., Zheng, C., Jiang, M., Cao, G., Yang, Q., et al. (2016). Fate decision of mesenchymal stem cells: adipocytes or osteoblasts? Cell Death Differ. 23, 1128-1139. doi: 10.1038/cdd.2015.168

Cho, Y. M., Kwon, S., Pak, Y. K., Seol, H. W., Choi, Y. M., Park, D. J., et al. (2006). Dynamic changes in mitochondrial biogenesis and antioxidant enzymes during the spontaneous differentiation of human embryonic stem cells. Biochem. Biophys. Res. Commun. 348, 1472-1478. doi: 10.1016/j.bbrc.2006. 08.020

Cogliati, S., Frezza, C., Soriano, M. E., Varanita, T., Quintana-Cabrera, R., Corrado, M., et al. (2013). Mitochondrial cristae shape determines respiratory chain supercomplexes assembly and respiratory efficiency. Cell 155, 160-171. doi: 10.1016/j.cell.2013.08.032

Collins, T. J., Berridge, M. J., Lipp, P., and Bootman, M. D. (2002). Mitochondria are morphologically and functionally heterogeneous within cells. EMBO J. 21, 1616-1627. doi: 10.1093/emboj/21.7.1616

Cook, S. J., Stuart, K., Gilley, R., and Sale, M. J. (2017). Control of cell death and mitochondrial fission by ERK1/2 MAP kinase signalling. FEBS J. 284, 4177-4195. doi: 10.1111/febs.14122

Cribbs, J. T., and Strack, S. (2007). Reversible phosphorylation of Drp1 by cyclic AMP-dependent protein kinase and calcineurin regulates mitochondrial fission and cell death. EMBO Rep. 8, 939-944. doi: 10.1038/sj.embor.7401062

D’Amico, D., Mottis, A., Potenza, F., Sorrentino, V., Li, H., Romani, M., et al. (2019). The RNA-Binding protein PUM2 impairs mitochondrial dynamics and mitophagy during aging. Mol. Cell 73, 775.e-787.e. doi: 10.1016/j.molcel.2018. 11.034

Deng, R., Liu, Y., He, H., Zhang, H., Zhao, C., Cui, Z., et al. (2020). Haemin pre-treatment augments the cardiac protection of mesenchymal stem cells by inhibiting mitochondrial fission and improving survival. J. Cell Mol. Med. 24, 431-440. doi: 10.1111/jcmm.14747

Dilberger, B., Baumanns, S., Schmitt, F., Schmiedl, T., Hardt, M., Wenzel, U., et al. (2019). Mitochondrial oxidative stress impairs energy metabolism and reduces stress resistance and longevity of c. elegans. Oxid. Med. Cell Longev. 2019:6840540. doi: 10.1155/2019/6840540

Ding, M., Dong, Q., Liu, Z., Liu, Z., Qu, Y., Li, X., et al. (2017). Inhibition of dynamin-related protein 1 protects against myocardial ischemia-reperfusion injury in diabetic mice. Cardiovasc. Diabetol. 16:19. doi: 10.1186/s12933-0170501-502

Disatnik, M. H., Ferreira, J. C., Campos, J. C., Gomes, K. S., Dourado, P. M., Qi, X., et al. (2013). Acute inhibition of excessive mitochondrial fission after myocardial infarction prevents long-term cardiac dysfunction. J. Am. Heart Assoc. 2:e000461. doi: 10.1161/jaha.113.000461

Ducommun, S., Deak, M., Sumpton, D., Ford, R. J., Núñez Galindo, A., Kussmann, M., et al. (2015). Motif affinity and mass spectrometry proteomic approach for the discovery of cellular AMPK targets: identification of mitochondrial fission factor as a new AMPK substrate. Cell Signal 27, 978-988. doi: 10.1016/j.cellsig. 2015.02.008

Egan, D. F., Shackelford, D. B., Mihaylova, M. M., Gelino, S., Kohnz, R. A., Mair, W., et al. (2011). Phosphorylation of ULK1 (hATG1) by AMP-Activated Protein Kinase Connects Energy Sensing to Mitophagy. Science 331, 456-461. doi: 10.1126/science.1196371

Eisner, V., Picard, M., and Hajnóczky, G. (2018). Mitochondrial dynamics in adaptive and maladaptive cellular stress responses. Nat. Cell Biol. 20, 755-765. doi: 10.1038/s41556-018-0133-130

El-Hattab, A. W., Suleiman, J., Almannai, M., and Scaglia, F. (2018). Mitochondrial dynamics: biological roles, molecular machinery, and related diseases. Mol. Genet. Metab 125, 315-321. doi: 10.1016/j.ymgme.2018.10.003

Fan, L., Wang, J., and Ma, C. (2019). Pretreatment of bone mesenchymal stem cells with miR181-c facilitates craniofacial defect reconstruction via activating AMPK-Mfn1 signaling pathways. J. Recept. Signal Transduct. Res. 39, 199-207. doi: 10.1080/10799893.2019.1652649

Fang, D., Yan, S., Yu, Q., Chen, D., and Yan, S. S. (2016). Mfn2 is Required for Mitochondrial Development and Synapse Formation in Human Induced Pluripotent Stem Cells/hiPSC Derived Cortical Neurons. Sci. Rep. 6:31462. doi: 10.1038/srep31462

Farahani, R. A., Farah, M. C., Zhu, X. Y., Tang, H., Saadiq, I. M., Lerman, L. O., et al. (2020). Metabolic syndrome impairs 3D Mitochondrial structure, dynamics, and function in swine mesenchymal stem cells. Stem Cell Rev. Rep. 16, 933-945. doi: 10.1007/s12015-020-09988-9983

Feliciello, A., Gottesman, M. E., and Avvedimento, E. V. (2005). cAMP-PKA signaling to the mitochondria: protein scaffolds, mRNA and phosphatases. Cell Signal 17, 279-287. doi: 10.1016/j.cellsig.2004.09.009

Feng, X., Zhang, W., Yin, W., and Kang, Y. J. (2019). Feature article: the involvement of mitochondrial fission in maintenance of the stemness of bone marrow mesenchymal stem cells. Exp. Biol. Med. (Maywood) 244, 64-72. doi: $10.1177 / 1535370218821063$

Ferreira, J. C. B., Campos, J. C., Qvit, N., Qi, X., Bozi, L. H. M., Bechara, L. R. G., et al. (2019). A selective inhibitor of mitofusin 1- $\beta$ IIPKC association improves heart failure outcome in rats. Nat. Commun. 10:329. doi: 10.1038/s41467-01808276-8276

Fillmore, N., Huqi, A., Jaswal, J. S., Mori, J., Paulin, R., Haromy, A., et al. (2015). Effect of fatty acids on human bone marrow mesenchymal stem cell energy metabolism and survival. PLoS One 10:e0120257. doi: 10.1371/journal.pone. 0120257

Folmes, C. D., Nelson, T. J., Martinez-Fernandez, A., Arrell, D. K., Lindor, J. Z., Dzeja, P. P., et al. (2011). Somatic oxidative bioenergetics transitions into pluripotency-dependent glycolysis to facilitate nuclear reprogramming. Cell Metab 14, 264-271. doi: 10.1016/j.cmet.2011.06.011

Forni, M. F., Peloggia, J., Trudeau, K., Shirihai, O., and Kowaltowski, A. J. (2016). Murine mesenchymal stem cell commitment to differentiation is regulated by mitochondrial dynamics. Stem Cells 34, 743-755. doi: 10.1002/stem.2248

Frank, M., Duvezin-Caubet, S., Koob, S., Occhipinti, A., Jagasia, R., Petcherski, A., et al. (2012). Mitophagy is triggered by mild oxidative stress in a mitochondrial fission dependent manner. Biochim. Biophys. Acta 1823, 2297-2310. doi: 10. 1016/j.bbamcr.2012.08.007

Fu, L., Dong, Q., He, J., Wang, X., Xing, J., Wang, E., et al. (2017). SIRT4 inhibits malignancy progression of NSCLCs, through mitochondrial dynamics mediated by the ERK-Drpl pathway. Oncogene 36, 2724-2736. doi: 10.1038/ onc. 2016.425

Fu, W. Y., Liu, Y., and Yin, H. (2019). Mitochondrial dynamics: biogenesis, fission, fusion, and mitophagy in the regulation of stem cell behaviors. Stem Cells Int. 2019:9757201. doi: 10.1155/2019/9757201

Fujiwara, M., Tian, L., Le, P. T., DeMambro, V. E., Becker, K. A., Rosen, C. J., et al. (2019). The mitophagy receptor Bcl-2-like protein 13 stimulates adipogenesis by regulating mitochondrial oxidative phosphorylation and apoptosis in mice. J. Biol. Chem. 294, 12683-12694. doi: 10.1074/jbc.RA119.008630

Gao, D., Zhang, L., Dhillon, R., Hong, T. T., Shaw, R. M., and Zhu, J. (2013). Dynasore protects mitochondria and improves cardiac lusitropy in Langendorff perfused mouse heart. PLoS One 8:e60967. doi: 10.1371/journal.pone.0060967

Geissler, S., Textor, M., Kühnisch, J., Könnig, D., Klein, O., Ode, A., et al. (2012). Functional comparison of chronological and in vitro aging: differential role of the cytoskeleton and mitochondria in mesenchymal stromal cells. PLoS One 7:e52700. doi: 10.1371/journal.pone.0052700 
Gilkerson, R. W., Selker, J. M. L., and Capaldi, R. A. (2003). The cristal membrane of mitochondria is the principal site of oxidative phosphorylation. Febs Lett. 546, 355-358. doi: 10.1016/S0014-5793(03)00633-631

Gomes, L. C., Di Benedetto, G., and Scorrano, L. (2011). During autophagy mitochondria elongate, are spared from degradation and sustain cell viability. Nat. Cell Biol. 13, 589-598. doi: 10.1038/ncb2220

Gomes, L. C., and Scorrano, L. (2008). High levels of Fis1, a pro-fission mitochondrial protein, trigger autophagy. Biochim. Biophys. Acta 1777, 860866. doi: 10.1016/j.bbabio.2008.05.442

Guedouari, H., Daigle, T., Scorrano, L., and Hebert-Chatelain, E. (2017). Sirtuin 5 protects mitochondria from fragmentation and degradation during starvation. Biochim. Biophys. Acta Mol. Cell Res. 1864, 169-176. doi: 10.1016/j.bbamcr. 2016.10.015

Guo, J., Ren, R., Yao, X., Ye, Y., Sun, K., Lin, J., et al. (2020). PKM2 suppresses osteogenesis and facilitates adipogenesis by regulating $\beta$-catenin signaling and mitochondrial fusion and fission. Aging (Albany NY) 12, 3976-3992. doi: 10. 18632/aging.102866

Han, Y. S., Kim, S. M., Lee, J. H., Jung, S. K., Noh, H., and Lee, S. H. (2019). Melatonin protects chronic kidney disease mesenchymal stem cells against senescence via $\operatorname{PrP}(C)$-dependent enhancement of the mitochondrial function. J. Pineal. Res. 66:e12535. doi: 10.1111/jpi.12535

Hawkins, K. E., Joy, S., Delhove, J. M. K. M., Kotiadis, V. N., Fernandez, E., Fitzpatrick, L. M., et al. (2016). NRF2 Orchestrates the Metabolic Shift during Induced Pluripotent Stem Cell Reprogramming. Cell Rep. 14, 1883-1891. doi: 10.1016/j.celrep.2016.02.003

He, H., Yu, B., Liu, Z., Ye, G., You, W., Hong, Y., et al. (2019). Vascular progenitor cell senescence in patients with Marfan syndrome. J. Cell Mol. Med. 23, 41394152. doi: $10.1111 /$ jcmm.14301

He, Y., Gan, X., Zhang, L., Liu, B., Zhu, Z., Li, T., et al. (2018). CoCl(2) induces apoptosis via a ROS-dependent pathway and Drp1-mediated mitochondria fission in periodontal ligament stem cells. Am. J. Physiol. Cell Physiol. 315, C389-C397. doi: 10.1152/ajpcell.00248.2017

Hong, Y., He, H., Jiang, G., Zhang, H., Tao, W., Ding, Y., et al. (2020). miR$155-5 \mathrm{p}$ inhibition rejuvenates aged mesenchymal stem cells and enhances cardioprotection following infarction. Aging Cell 19:e13128. doi: 10.1111/acel. 13128

Hoppins, S., Lackner, L., and Nunnari, J. (2007). The machines that divide and fuse mitochondria. Ann. Rev. Biochem. 76, 751-780. doi: 10.1146/annurev.biochem. 76.071905.090048

Hoque, A., Sivakumaran, P., Bond, S. T., Ling, N. X. Y., Kong, A. M., Scott, J. W., et al. (2018). Mitochondrial fission protein Drp1 inhibition promotes cardiac mesodermal differentiation of human pluripotent stem cells. Cell Death Discov. 4:39. doi: 10.1038/s41420-018-0042-49

Hsu, Y. C., Wu, Y. T., Yu, T. H., and Wei, Y. H. (2016). Mitochondria in mesenchymal stem cell biology and cell therapy: from cellular differentiation to mitochondrial transfer. Semin Cell Dev. Biol. 52, 119-131. doi: 10.1016/j. semcdb.2016.02.011

Hu, C., Zhao, L., Peng, C., and Li, L. (2018a). Regulation of the mitochondrial reactive oxygen species: strategies to control mesenchymal stem cell fates ex vivo and in vivo. J. Cell Mol. Med. 22, 5196-5207. doi: 10.1111/jcmm.13835

Hu, L., Yin, C., Zhao, F., Ali, A., Ma, J., and Qian, A. (2018b). Mesenchymal stem cells: cell fate decision to osteoblast or adipocyte and application in osteoporosis treatment. Int. J. Mol. Sci. 19:360. doi: 10.3390/ijms19020360

Huang, J. Y., Hirschey, M. D., Shimazu, T., Ho, L., and Verdin, E. (2010). Mitochondrial sirtuins. Biochim. Biophys. Acta 1804, 1645-1651. doi: 10.1016/j. bbapap.2009.12.021

Hye Kim, J., Gyu Park, S., Kim, W. K., Song, S. U., and Sung, J. H. (2015). Functional regulation of adipose-derived stem cells by PDGF-D. Stem Cells 33, 542-556. doi: 10.1002/stem.1865

Ikeda, Y., Shirakabe, A., Brady, C., Zablocki, D., Ohishi, M., and Sadoshima, J. (2015). Molecular mechanisms mediating mitochondrial dynamics and mitophagy and their functional roles in the cardiovascular system. J. Mol. Cell Cardiol. 78, 116-122. doi: 10.1016/j.yjmcc.2014. 09.019

Jahani-Asl, A., and Slack, R. S. (2007). The phosphorylation state of Drp1 determines cell fate. EMBO Rep. 8, 912-913. doi: 10.1038/sj.embor.7401077

Jendrach, M., Mai, S., Pohl, S., Vöth, M., and Bereiter-Hahn, J. (2008). Shortand long-term alterations of mitochondrial morphology, dynamics and mtDNA after transient oxidative stress. Mitochondrion 8, 293-304. doi: 10.1016/j.mito. 2008.06.001

Ježek, J., Cooper, K. F., and Strich, R. (2018). Reactive oxygen species and mitochondrial dynamics: the yin and yang of mitochondrial dysfunction and cancer progression. Antioxidants (Basel) 7:13. doi: 10.3390/antiox7010013

Jheng, H. F., Huang, S. H., Kuo, H. M., Hughes, M. W., and Tsai, Y. S. (2015). Molecular insight and pharmacological approaches targeting mitochondrial dynamics in skeletal muscle during obesity. Ann. N. Y. Acad. Sci. 1350, 82-94. doi: $10.1111 /$ nyas. 12863

Jheng, H. F., Tsai, P. J., Guo, S. M., Kuo, L. H., Chang, C. S., Su, I. J., et al. (2012). Mitochondrial fission contributes to mitochondrial dysfunction and insulin resistance in skeletal muscle. Mol. Cell Biol. 32, 309-319. doi: 10.1128/mcb. 05603-5611

Jin, X., Su, R., Li, R., Cheng, L., and Li, Z. (2018). Crucial role of pro-inflammatory cytokines from respiratory tract upon $\mathrm{PM}(2.5)$ exposure in causing the BMSCs differentiation in cells and animals. Oncotarget 9, 1745-1759. doi: 10.18632/ oncotarget. 23158

Kang, S. W., Haydar, G., Taniane, C., Farrell, G., Arias, I. M., LippincottSchwartz, J., et al. (2016). AMPK Activation prevents and reverses drug-induced mitochondrial and hepatocyte injury by promoting mitochondrial fusion and function. PLoS One 11:e0165638. doi: 10.1371/journal.pone.0165638

Kasahara, A., Cipolat, S., Chen, Y., Dorn, G. W. II, and Scorrano, L. (2013). Mitochondrial fusion directs cardiomyocyte differentiation via calcineurin and Notch signaling. Science 342, 734-737. doi: 10.1126/science.1241359

Kashatus, J. A., Nascimento, A., Myers, L. J., Sher, A., Byrne, F. L., Hoehn, K. L., et al. (2015). Erk2 phosphorylation of Drp1 promotes mitochondrial fission and MAPK-driven tumor growth. Mol. Cell 57, 537-551. doi: 10.1016/j.molcel.2015. 01.002

Kasper, G., Mao, L., Geissler, S., Draycheva, A., Trippens, J., Kuhnisch, J., et al. (2009). Insights into mesenchymal stem cell aging: involvement of antioxidant defense and actin cytoskeleton. Stem Cells 27, 1288-1297. doi: 10.1002/stem.49

Khacho, M., Clark, A., Svoboda, D. S., Azzi, J., MacLaurin, J. G., Meghaizel, C., et al. (2016). Mitochondrial dynamics impacts stem cell identity and fate decisions by regulating a nuclear transcriptional program. Cell Stem Cell 19, 232-247. doi: 10.1016/j.stem.2016.04.015

Khacho, M., Tarabay, M., Patten, D., Khacho, P., MacLaurin, J. G., Guadagno, J., et al. (2014). Acidosis overrides oxygen deprivation to maintain mitochondrial function and cell survival. Nat. Commun. 5:3550. doi: 10.1038/ncomms 4550

Ko, E., Lee, K. Y., and Hwang, D. S. (2012). Human umbilical cord blood-derived mesenchymal stem cells undergo cellular senescence in response to oxidative stress. Stem Cells Dev. 21, 1877-1886. doi: 10.1089/scd.2011.0284

Ko, S. H., Choi, G. E., Oh, J. Y., Lee, H. J., Kim, J. S., Chae, C. W., et al. (2017). Succinate promotes stem cell migration through the GPR91-dependent regulation of DRP1-mediated mitochondrial fission. Sci. Rep. 7:12582. doi: 10. 1038/s41598-017-12692-x

Kornicka, K., Szłapka-Kosarzewska, J., Śmieszek, A., and Marycz, K. (2019). 5Azacytydine and resveratrol reverse senescence and ageing of adipose stem cells via modulation of mitochondrial dynamics and autophagy. J. Cell Mol. Med. 23, 237-259. doi: 10.1111/jcmm.13914

Kwon, H. J., and Ohmiya, Y. (2013). Metabolomic analysis of differential changes in metabolites during ATP oscillations in chondrogenesis. Biomed. Res. Int. 2013:213972. doi: 10.1155/2013/213972

Lambertini, E., Penolazzi, L., Morganti, C., Lisignoli, G., Zini, N., Angelozzi, M., et al. (2015). Osteogenic differentiation of human MSCs: specific occupancy of the mitochondrial DNA by NFATc1 transcription factor. Int. J. Biochem. Cell Biol. 64, 212-219. doi: 10.1016/j.biocel.2015.04.011

Lang, A., Anand, R., Altinoluk-Hambüchen, S., Ezzahoini, H., Stefanski, A., Iram, A., et al. (2017). SIRT4 interacts with OPA1 and regulates mitochondrial quality control and mitophagy. Aging (Albany NY) 9, 2163-2189. doi: 10.18632/aging. 101307

Leboucher, G. P., Tsai, Y. C., Yang, M., Shaw, K. C., Zhou, M., Veenstra, T. D., et al. (2012). Stress-induced phosphorylation and proteasomal degradation of mitofusin 2 facilitates mitochondrial fragmentation and apoptosis. Mol. Cell 47, 547-557. doi: 10.1016/j.molcel.2012.05.041

Li, C. J., Chen, P. K., Sun, L. Y., and Pang, C. Y. (2017a). Enhancement of mitochondrial transfer by antioxidants in human mesenchymal stem cells. Oxid. Med. Cell Longev. 2017:8510805. doi: 10.1155/2017/8510805 
Li, Q., Gao, Z., Chen, Y., and Guan, M. X. (2017b). The role of mitochondria in osteogenic, adipogenic and chondrogenic differentiation of mesenchymal stem cells. Protein Cell 8, 439-445. doi: 10.1007/s13238-017-0385-387

Li, C. J., Sun, L. Y., and Pang, C. Y. (2015). Synergistic protection of $\mathrm{N}$-acetylcysteine and ascorbic acid 2-phosphate on human mesenchymal stem cells against mitoptosis, necroptosis and apoptosis. Sci. Rep. 5:9819. doi: 10. 1038/srep09819

Li, J., Liu, X. Y., Zuo, B., and Zhang, L. (2016). The role of bone marrow microenvironment in governing the balance between osteoblastogenesis and adipogenesis. Aging Dis. 7, 514-525. doi: 10.14336/Ad.2015.1206

Li, X., Hong, Y., He, H., Jiang, G., You, W., Liang, X., et al. (2019). FGF21 mediates mesenchymal stem cell senescence via regulation of mitochondrial dynamics. Oxid. Med. Cell Longev. 2019:4915149. doi: 10.1155/2019/4915149

Liang, R., Arif, T., Kalmykova, S., Kasianov, A., Lin, M., Menon, V., et al. (2020). Restraining lysosomal activity preserves hematopoietic stem cell quiescence and potency. Cell Stem Cell 26, 359-376.e7. doi: 10.1016/j.stem.2020.01.013

Lin, J. R., Shen, W. L., Yan, C., and Gao, P. J. (2015). Downregulation of dynaminrelated protein 1 contributes to impaired autophagic flux and angiogenic function in senescent endothelial cells. Arterioscler Thromb Vasc. Biol. 35, 1413-1422. doi: 10.1161/atvbaha.115.305706

Lin, W., Xu, L., Pan, Q., Lin, S., Feng, L., Wang, B., et al. (2019). Lgr5overexpressing mesenchymal stem cells augment fracture healing through regulation of Wnt/ERK signaling pathways and mitochondrial dynamics. FASEB J. 33, 8565-8577. doi: 10.1096/fj.201900082RR

Lisowski, P., Kannan, P., Mlody, B., and Prigione, A. (2018). Mitochondria and the dynamic control of stem cell homeostasis. EMBO Rep. 19:e45432. doi: $10.15252 / \mathrm{embr} .201745432$

Lorenz, C., Lesimple, P., Bukowiecki, R., Zink, A., Inak, G., Mlody, B., et al. (2017). Human iPSC-Derived neural progenitors are an effective drug discovery model for neurological mtDNA disorders. Cell Stem Cell 20, 659-674.e9. doi: 10.1016/j.stem.2016.12.013

Losón, O. C., Song, Z., Chen, H., and Chan, D. C. (2013). Fis1, Mff, MiD49, and MiD51 mediate Drp1 recruitment in mitochondrial fission. Mol. Biol. Cell 24, 659-667. doi: 10.1091/mbc.E12-10-0721

Luchsinger, L. L., de Almeida, M. J., Corrigan, D. J., Mumau, M., and Snoeck, H. W. (2016). Mitofusin 2 maintains haematopoietic stem cells with extensive lymphoid potential. Nature 529, 528-531. doi: 10.1038/nature16500

Ma, L., Feng, X., Wang, K., Song, Y., Luo, R., and Yang, C. (2019). Dexamethasone promotes mesenchymal stem cell apoptosis and inhibits osteogenesis by disrupting mitochondrial dynamics. FEBS Open Biol. 10, 211-220. doi: 10.1002/ 2211-5463.12771

MacVicar, T., and Langer, T. (2016). OPA1 processing in cell death and disease the long and short of it. J. Cell Sci. 129, 2297-2306. doi: 10.1242/jcs.159186

Mai, S., Klinkenberg, M., Auburger, G., Bereiter-Hahn, J., and Jendrach, M. (2010). Decreased expression of Drp1 and Fis1 mediates mitochondrial elongation in senescent cells and enhances resistance to oxidative stress through PINK1. J. Cell Sci. 123(Pt 6), 917-926. doi: 10.1242/jcs.059246

Marycz, K., Houston, J. M. I., Weiss, C., Rocken, M., and Kornicka, K. (2019). 5-Azacytidine and resveratrol enhance chondrogenic differentiation of metabolic syndrome-derived mesenchymal stem cells by modulating autophagy. Oxid. Med. Cell Longev. 2019:1523140. doi: 10.1155/2019/ 1523140

Marycz, K., Kornicka, K., Grzesiak, J., Śmieszek, A., and Szłapka, J. (2016). Macroautophagy and selective mitophagy ameliorate chondrogenic differentiation potential in adipose stem cells of equine metabolic syndrome: new findings in the field of progenitor cells differentiation. Oxid. Med. Cell Longev. 2016:3718468. doi: 10.1155/2016/3718468

Marycz, K., Kornicka, K., Irwin-Houston, J. M., and Weiss, C. (2018). Combination of resveratrol and 5-azacytydine improves osteogenesis of metabolic syndrome mesenchymal stem cells. J. Cell Mol. Med. 22, 4771-4793. doi: 10.1111/jcmm. 13731

Merz, S., Hammermeister, M., Altmann, K., Dürr, M., and Westermann, B. (2007). Molecular machinery of mitochondrial dynamics in yeast. Biol. Chem. 388, 917-926. doi: 10.1515/bc.2007.110

Meyer, J. N., Leuthner, T. C., and Luz, A. L. (2017). Mitochondrial fusion, fission, and mitochondrial toxicity. Toxicology 391, 42-53. doi: 10.1016/j.tox.2017. 07.019
Molina, A. J., Wikstrom, J. D., Stiles, L., Las, G., Mohamed, H., Elorza, A., et al. (2009). Mitochondrial networking protects beta-cells from nutrient-induced apoptosis. Diabetes 58, 2303-2315. doi: 10.2337/db07-1781

Muruganandan, S., Ionescu, A. M., and Sinal, C. J. (2020). At the crossroads of the adipocyte and osteoclast differentiation programs: future therapeutic perspectives. Int. J. Mol. Sci. 21:2277. doi: 10.3390/ijms21072277

Palmeira, C. M., Teodoro, J. S., Amorim, J. A., Steegborn, C., Sinclair, D. A., and Rolo, A. P. (2019). Mitohormesis and metabolic health: the interplay between ROS, cAMP and sirtuins. Free Radic Biol. Med. 141, 483-491. doi: 10.1016/j. freeradbiomed.2019.07.017

Papa, L., Djedaini, M., and Hoffman, R. (2019). Mitochondrial role in stemness and differentiation of hematopoietic stem cells. Stem Cells Int. 2019:4067162. doi: $10.1155 / 2019 / 4067162$

Papa, L., Zimran, E., Djedaini, M., Ge, Y., Ozbek, U., Sebra, R., et al. (2018). Ex vivo human HSC expansion requires coordination of cellular reprogramming with mitochondrial remodeling and p53 activation. Blood Adv. 2, 2766-2779. doi: 10.1182/bloodadvances.2018024273

Park, S. J., Lee, S. B., Suh, Y., Kim, S. J., Lee, N., Hong, J. H., et al. (2017). DISC1 modulates neuronal stress responses by gate-keeping ER-mitochondria $\mathrm{Ca} 2+$ Transfer through the MAM. Cell Rep. 21, 2748-2759. doi: 10.1016/j.celrep.2017. 11.043

Patten, D. A., Ouellet, M., Allan, D. S., Germain, M., Baird, S. D., Harper, M. E., et al. (2019). Mitochondrial adaptation in human mesenchymal stem cells following ionizing radiation. FASEB J. 33, 9263-9278. doi: 10.1096/fj. 201801483RR

Polletta, L., Vernucci, E., Carnevale, I., Arcangeli, T., Rotili, D., Palmerio, S., et al. (2015). SIRT5 regulation of ammonia-induced autophagy and mitophagy. Autophagy 11, 253-270. doi: 10.1080/15548627.2015.1009778

Prieto, J., Leon, M., Ponsoda, X., Sendra, R., Bort, R., Ferrer-Lorente, R., et al. (2016). Early ERK1/2 activation promotes DRP1-dependent mitochondrial fission necessary for cell reprogramming. Nat. Commun. 7:11124. doi: 10.1038/ ncomms 11124

Pyakurel, A., Savoia, C., Hess, D., and Scorrano, L. (2015). Extracellular regulated kinase phosphorylates mitofusin 1 to control mitochondrial morphology and apoptosis. Mol. Cell 58, 244-254. doi: 10.1016/j.molcel.2015.02.021

Qi, M., Zhang, L., Ma, Y., Shuai, Y., Li, L., Luo, K., et al. (2017). Autophagy maintains the function of bone marrow mesenchymal stem cells to prevent estrogen deficiency-induced osteoporosis. Theranostics 7, 4498-4516. doi: 10. 7150/thno. 17949

Rajabzadeh, N., Fathi, E., and Farahzadi, R. (2019). Stem cell-based regenerative medicine. Stem Cell Investig. 6:19. doi: 10.21037/sci.2019.06.04

Ribeiro, M. F., Genebra, T., Rego, A. C., Rodrigues, C. M. P., and Solá, S. (2019). Amyloid $\beta$ peptide compromises neural stem cell fate by irreversibly disturbing mitochondrial oxidative state and blocking mitochondrial biogenesis and dynamics. Mol. Neurobiol. 56, 3922-3936. doi: 10.1007/s12035-018-1342-z

Romero-Moya, D., Bueno, C., Montes, R., Navarro-Montero, O., Iborra, F. J., Lopez, L. C., et al. (2013). Cord blood-derived CD34+ hematopoietic cells with low mitochondrial mass are enriched in hematopoietic repopulating stem cell function. Haematologica 98, 1022-1029. doi: 10.3324/haematol.2012.079244

Rosdah, A. A., Bond, S. T., Sivakumaran, P., Hoque, A., Oakhill, J. S., Drew, B. G., et al. (2017). Mdivi-1 Protects Human W8B2(+) Cardiac Stem Cells from Oxidative Stress and Simulated Ischemia-Reperfusion Injury. Stem Cells Dev. 26, 1771-1780. doi: 10.1089/scd.2017.0157

Samant, S. A., Zhang, H. J., Hong, Z., Pillai, V. B., Sundaresan, N. R., Wolfgeher, D., et al. (2014). SIRT3 deacetylates and activates OPA1 to regulate mitochondrial dynamics during stress. Mol. Cell Biol. 34, 807-819. doi: 10.1128/mcb.014831413

Santel, A., and Fuller, M. T. (2001). Control of mitochondrial morphology by a human mitofusin. J. Cell Sci. 114(Pt 5), 867-874.

Senos Demarco, R., Uyemura, B. S., D’Alterio, C., and Jones, D. L. (2019). Mitochondrial fusion regulates lipid homeostasis and stem cell maintenance in the Drosophila testis. Nat. Cell Biol. 21, 710-720. doi: 10.1038/s41556-0190332-333

Seo, B. J., Yoon, S. H., and Do, J. T. (2018). Mitochondrial dynamics in stem cells and differentiation. Int. J. Mol. Sci. 19:3893. doi: 10.3390/ijms19123893

Serasinghe, M. N., Wieder, S. Y., Renault, T. T., Elkholi, R., Asciolla, J. J., Yao, J. L., et al. (2015). Mitochondrial division is requisite to RAS-induced transformation 
and targeted by oncogenic MAPK pathway inhibitors. Mol. Cell 57, 521-536. doi: 10.1016/j.molcel.2015.01.003

Shadel, G. S., and Horvath, T. L. (2015). Mitochondrial ROS signaling in organismal homeostasis. Cell 163, 560-569. doi: 10.1016/j.cell.2015.10.001

Shaughnessy, D. T., McAllister, K., Worth, L., Haugen, A. C., Meyer, J. N., Domann, F. E., et al. (2014). Mitochondria, energetics, epigenetics, and cellular responses to stress. Environ. Health Perspect. 122, 1271-1278. doi: 10.1289/ehp.140 8418

Shen, Y., Wu, L., Wang, J., Wu, X., and Zhang, X. (2018a). The role of mitochondria in methamphetamine-induced inhibitory effects on osteogenesis of mesenchymal stem cells. Eur. J. Pharmacol. 826, 56-65. doi: 10.1016/j.ejphar. 2018.02.049

Shen, Y. L., Wu, L., Qin, D. D., Xia, Y. K., Zhou, Z., Zhang, X. M., et al. (2018b). Carbon black suppresses the osteogenesis of mesenchymal stem cells: the role of mitochondria. Part. Fibre Toxicol. 15:16. doi: 10.1186/s12989-018-0253-255

Signorile, A., Santeramo, A., Tamma, G., Pellegrino, T., D’Oria, S., Lattanzio, P., et al. (2017). Mitochondrial cAMP prevents apoptosis modulating Sirt3 protein level and OPA1 processing in cardiac myoblast cells. Biochim. Biophys. Acta Mol. Cell Res. 1864, 355-366. doi: 10.1016/j.bbamcr.2016.11.022

Sisakhtnezhad, S., Alimoradi, E., and Akrami, H. (2017). External factors influencing mesenchymal stem cell fate in vitro. Eur. J. Cell Biol. 96, 13-33. doi: $10.1016 /$ j.ejcb.2016.11.003

Son, M. J., Kwon, Y., Son, M. Y., Seol, B., Choi, H. S., Ryu, S. W., et al. (2015). Mitofusins deficiency elicits mitochondrial metabolic reprogramming to pluripotency. Cell Death Differ. 22, 1957-1969. doi: 10.1038/cdd.2015.43

Sperber, H., Mathieu, J., Wang, Y., Ferreccio, A., Hesson, J., Xu, Z., et al. (2015). The metabolome regulates the epigenetic landscape during naive-to-primed human embryonic stem cell transition. Nat. Cell Biol. 17, 1523-1535. doi: 10. 1038/ncb3264

Srivastava, A., Singh, S., Rajpurohit, C. S., Srivastava, P., Pandey, A., Kumar, D., et al. (2018). Secretome of differentiated PC12 cells restores the monocrotophos-induced damages in human mesenchymal stem cells and SHSY-5Y cells: role of autophagy and mitochondrial dynamics. Neuromol. Med. 20, 233-251. doi: 10.1007/s12017-018-8487-8489

Stab, B. R. II, Martinez, L., Grismaldo, A., Lerma, A., Gutiérrez, M. L., Barrera, L. A., et al. (2016). Mitochondrial functional changes characterization in young and senescent human adipose derived MSCs. Front. Aging Neurosci. 8:299. doi: 10.3389/fnagi.2016.00299

Stiles, L., and Shirihai, O. S. (2012). Mitochondrial dynamics and morphology in beta-cells. Best Pract. Res. Clin. Endocrinol. Metab 26, 725-738. doi: 10.1016/j. beem.2012.05.004

Strauss, M., Hofhaus, G., Schroeder, R. R., and Kuhlbrandt, W. (2008). Dimer ribbons of ATP synthase shape the inner mitochondrial membrane. EMBO J. 27, 1154-1160. doi: 10.1038/emboj.2008.35

Sugioka, R., Shimizu, S., and Tsujimoto, Y. (2004). Fzol, a protein involved in mitochondrial fusion, inhibits apoptosis. J. Biol. Chem. 279, 52726-52734. doi: 10.1074/jbc.M408910200

Szabadkai, G., Simoni, A. M., Chami, M., Wieckowski, M. R., Youle, R. J., and Rizzuto, R. (2004). Drp-1-dependent division of the mitochondrial network blocks intraorganellar $\mathrm{Ca} 2+$ waves and protects against $\mathrm{Ca} 2+$-mediated apoptosis. Mol. Cell 16, 59-68. doi: 10.1016/j.molcel.2004.09.026

Tan, D. Q., and Suda, T. (2018). Reactive oxygen species and mitochondrial homeostasis as regulators of stem cell fate and function. Antioxid Redox Signal 29, 149-168. doi: 10.1089/ars.2017.7273

Tang, Y., Wu, X., Lei, W., Pang, L., Wan, C., Shi, Z., et al. (2009). TGF-beta1induced migration of bone mesenchymal stem cells couples bone resorption with formation. Nat. Med. 15, 757-765. doi: 10.1038/nm.1979

Tarte, K., Gaillard, J., Lataillade, J. J., Fouillard, L., Becker, M., Mossafa, H., et al. (2010). Clinical-grade production of human mesenchymal stromal cells: occurrence of aneuploidy without transformation. Blood 115, 1549-1553. doi: 10.1182/blood-2009-05-219907

Tondera, D., Grandemange, S., Jourdain, A., Karbowski, M., Mattenberger, Y., Herzig, S., et al. (2009). SLP-2 is required for stress-induced mitochondrial hyperfusion. EMBO J. 28, 1589-1600. doi: 10.1038/emboj.2009.89

Toyama, E. Q., Herzig, S., Courchet, J., Lewis, T. L. Jr., Losón, O. C., et al. (2016). Metabolism. AMP-activated protein kinase mediates mitochondrial fission in response to energy stress. Science 351, 275-281. doi: 10.1126/science.aab4138
Twig, G., Elorza, A., Molina, A. J. A., Mohamed, H., Wikstrom, J. D., Walzer, G., et al. (2008a). Fission and selective fusion govern mitochondrial segregation and elimination by autophagy. EMBO J. 27, 433-446. doi: 10.1038/sj.emboj. 7601963

Twig, G., Hyde, B., and Shirihai, O. S. (2008b). Mitochondrial fusion, fission and autophagy as a quality control axis: the bioenergetic view. Biochim. Biophys. Acta 1777, 1092-1097. doi: 10.1016/j.bbabio.2008.05.001

Vazquez-Martin, A., Cufi, S., Corominas-Faja, B., Oliveras-Ferraros, C., Vellon, L., and Menendez, J. A. (2012). Mitochondrial fusion by pharmacological manipulation impedes somatic cell reprogramming to pluripotency: new insight into the role of mitophagy in cell stemness. Aging-Us 4, 393-401. doi: 10.18632/aging. 100465

Vidoni, C., Ferraresi, A., Secomandi, E., Vallino, L., Gardin, C., Zavan, B., et al. (2019). Autophagy drives osteogenic differentiation of human gingival mesenchymal stem cells. Cell Commun. Signal 17:98. doi: 10.1186/s12964-0190414-417

Vina, J., Olaso-Gonzalez, G., Arc-Chagnaud, C., De la Rosa, A., and GomezCabrera, M. C. (2020). Modulating oxidant levels to promote healthy aging. Antioxid Redox Signal 33, 570-579. doi: 10.1089/ars.2020.8036

Wagner, W., Horn, P., Castoldi, M., Diehlmann, A., Bork, S., Saffrich, R., et al. (2008). Replicative senescence of mesenchymal stem cells: a continuous and organized process. PLoS One 3:e2213. doi: 10.1371/journal.pone.0002213

Wai, T., and Langer, T. (2016). Mitochondrial dynamics and metabolic regulation. Trends Endocrinol. Metab. 27, 105-117. doi: 10.1016/j.tem.2015.12.001

Wallace, D. C. (2005). A mitochondrial paradigm of metabolic and degenerative diseases, aging, and cancer: a dawn for evolutionary medicine. Ann. Rev. Genet. 39, 359-407. doi: 10.1146/annurev.genet.39.110304.095751

Wang, L., Zhang, T., Wang, L., Cai, Y., Zhong, X., He, X., et al. (2017a). Fatty acid synthesis is critical for stem cell pluripotency via promoting mitochondrial fission. $E M B O$ J. 36, 1330-1347. doi: 10.15252/embj.201695417

Wang, Q., Zhang, M., Torres, G., Wu, S., Ouyang, C., Xie, Z., et al. (2017b). Metformin suppresses diabetes-accelerated atherosclerosis via the inhibition of Drp1-Mediated mitochondrial fission. Diabetes 66, 193-205. doi: 10.2337/ db16-0915

Widmann, C., Gibson, S., Jarpe, M. B., and Johnson, G. L. (1999). Mitogenactivated protein kinase: conservation of a three-kinase module from yeast to human. Physiol. Rev. 79, 143-180. doi: 10.1152/physrev.1999.79.1.143

Wikstrom, J. D., Mahdaviani, K., Liesa, M., Sereda, S. B., Si, Y., Las, G., et al. (2014). Hormone-induced mitochondrial fission is utilized by brown adipocytes as an amplification pathway for energy expenditure. EMBO J. 33, 418-436. doi: $10.1002 / \mathrm{embj} .201385014$

Wu, J., Li, J., Chen, W. K., Liu, S., Liu, J. H., Zhang, J. S., et al. (2017). MicroRNA214 affects fibroblast differentiation of adipose-derived mesenchymal stem cells by targeting Mitofusin-2 during pelvic floor dysfunction in SD rats with birth trauma. Cell Physiol. Biochem. 42, 1870-1887. doi: 10.1159/000479570

Wu, J., Zhang, W., Ran, Q., Xiang, Y., Zhong, J. F., Li, S. C., et al. (2018). The differentiation balance of bone marrow mesenchymal stem cells is crucial to hematopoiesis. Stem Cells Int. 2018:1540148. doi: 10.1155/2018/1540148

Yang, F., Li, B., Yang, Y., Huang, M., Liu, X., Zhang, Y., et al. (2019). Leptin enhances glycolysis via OPA1-mediated mitochondrial fusion to promote mesenchymal stem cell survival. Int. J. Mol. Med. 44, 301-312. doi: 10.3892/ ijmm.2019.4189

Yang, F., Wu, R., Jiang, Z., Chen, J., Nan, J., Su, S., et al. (2018). Leptin increases mitochondrial OPA1 via GSK3-mediated OMA1 ubiquitination to enhance therapeutic effects of mesenchymal stem cell transplantation. Cell Death Dis. 9:556. doi: 10.1038/s41419-018-0579-579

Yao, X., Jing, X., Guo, J., Sun, K., Deng, Y., Zhang, Y., et al. (2019). Icariin protects bone marrow mesenchymal stem cells against iron overload induced dysfunction through mitochondrial fusion and fission, PI3K/AKT/mTOR and MAPK Pathways. Front. Pharmacol. 10:163. doi: 10.3389/fphar.2019.00163

Yi, X., Guo, W., Shi, Q., Yang, Y., Zhang, W., Chen, X., et al. (2019). SIRT3Dependent mitochondrial dynamics remodeling contributes to oxidative stressinduced melanocyte degeneration in vitiligo. Theranostics 9, 1614-1633. doi: 10.7150/thno. 30398

Yin, K., Zhu, R., Wang, S., and Zhao, R. C. (2017). Low-Level laser effect on proliferation, migration, and antiapoptosis of mesenchymal stem cells. Stem Cells Dev. 26, 762-775. doi: 10.1089/scd.2016.0332 
Yoon, Y. S., Yoon, D. S., Lim, I. K., Yoon, S. H., Chung, H. Y., Rojo, M., et al. (2006). Formation of elongated giant mitochondria in DFO-induced cellular senescence: involvement of enhanced fusion process through modulation of Fis1. J. Cell Physiol. 209, 468-480. doi: 10.1002/jcp.20753

Youle, R. J., and van der Bliek, A. M. (2012). Mitochondrial fission, fusion, and stress. Science 337, 1062-1065. doi: 10.1126/science.1219855

Yu, R., Liu, T., Ning, C., Tan, F., Jin, S. B., Lendahl, U., et al. (2019). The phosphorylation status of Ser-637 in dynamin-related protein 1 (Drp1) does not determine Drp1 recruitment to mitochondria. J. Biol. Chem. 294, 17262-17277. doi: 10.1074/jbc.RA119.008202

Yu, T., Robotham, J. L., and Yoon, Y. (2006). Increased production of reactive oxygen species in hyperglycemic conditions requires dynamic change of mitochondrial morphology. Proc. Natl. Acad. Sci. U.S.A. 103, 2653-2658. doi: $10.1073 /$ pnas.0511154103

Zhang, H. B., Menzies, K. J., and Auwerx, J. (2018). The role of mitochondria in stem cell fate and aging. Development 145:dev143420. doi: 10.1242/dev.14 3420

Zheng, Q., Zhao, Y., Guo, J., Zhao, S., Fei, C., Xiao, C., et al. (2018). Iron overload promotes mitochondrial fragmentation in mesenchymal stromal cells from myelodysplastic syndrome patients through activation of the AMPK/MFF/Drp1 pathway. Cell Death Dis. 9:515. doi: 10.1038/s41419-0180552-557

Zhong, X., Cui, P., Cai, Y., Wang, L., He, X., Long, P., et al. (2019). Mitochondrial Dynamics Is Critical for the Full Pluripotency and Embryonic Developmental Potential of Pluripotent Stem Cells. Cell Metab 29, 979-992.e4
Zhou, W., Chen, K. H., Cao, W., Zeng, J., Liao, H., Zhao, L., et al. (2010). Mutation of the protein kinase A phosphorylation site influences the antiproliferative activity of mitofusin 2. Atherosclerosis 211, 216-223. doi: 10.1016/ j.atherosclerosis.2010.02.012

Zhou, W., Choi, M., Margineantu, D., Margaretha, L., Hesson, J., Cavanaugh, C., et al. (2012). HIF1 $\alpha$ induced switch from bivalent to exclusively glycolytic metabolism during ESC-to-EpiSC/hESC transition. EMBO J. 31, 2103-2116. doi: 10.1038/emboj.2012.71

Zick, M., Rabl, R., and Reichert, A. S. (2009). Cristae formation-linking ultrastructure and function of mitochondria. Biochim. Biophys. Acta 1793, 5-19. doi: 10.1016/j.bbamcr.2008.06.013

Zorov, D. B., Juhaszova, M., and Sollott, S. J. (2014). Mitochondrial reactive oxygen species (ROS) and ROS-induced ROS release. Physiol. Rev. 94, 909-950. doi: 10.1152 /physrev.00026.2013

Conflict of Interest: The authors declare that the research was conducted in the absence of any commercial or financial relationships that could be construed as a potential conflict of interest.

Copyright (c) 2020 Ren, Chen, Chen, Li, Cheng and Xia. This is an open-access article distributed under the terms of the Creative Commons Attribution License (CC BY). The use, distribution or reproduction in other forums is permitted, provided the original author(s) and the copyright owner(s) are credited and that the original publication in this journal is cited, in accordance with accepted academic practice. No use, distribution or reproduction is permitted which does not comply with these terms. 